ESAIM: M2AN

Vol. 40, No 4, 2006, pp. 623-652

DOI: $10.1051 / \mathrm{m} 2 \mathrm{an}: 2006027$
ESAIM: Mathematical Modelling and Numerical Analysis

www.edpsciences.org/m2an

\title{
DIFFUSION AND PROPAGATION PROBLEMS IN SOME RAMIFIED DOMAINS WITH A FRACTAL BOUNDARY
}

\author{
Yves Achdou $^{1}$, Christophe Sabot ${ }^{2}$ and Nicoletta Tchou $^{3}$
}

\begin{abstract}
This paper is devoted to some elliptic boundary value problems in a self-similar ramified domain of $\mathbb{R}^{2}$ with a fractal boundary. Both the Laplace and Helmholtz equations are studied. A generalized Neumann boundary condition is imposed on the fractal boundary. Sobolev spaces on this domain are studied. In particular, extension and trace results are obtained. These results enable the investigation of the variational formulation of the above mentioned boundary value problems. Next, for homogeneous Neumann conditions, the emphasis is placed on transparent boundary conditions, which allow the computation of the solutions in the subdomains obtained by stopping the geometric construction after a finite number of steps. The proposed methods and algorithms will be used numerically in forecoming papers.
\end{abstract}

Mathematics Subject Classification. 28A80, 35J05, 35J25, 65N.

Received: September 26, 2005. Revised: March 1, 2006.

\section{INTRODUCTION}

In this paper, we deal with some boundary value problems in a self-similar ramified domain of $\mathbb{R}^{2}$ with a fractal boundary. This work was inspired by a wider and challenging project aimed at simulating the diffusion of medical sprays in lungs. Our goal are more modest here, since the geometry of the problems (only two dimensions) and the physical phenomena considered are much simpler, but we hope that rigorous results and methods will prove useful. We refer to $[5,15,16]$ for accurate physical descriptions of the lungs' physiology and for studies concerning the diffusion of oxygen in the lungs.

The geometry under consideration is that of a self-similar ramified bidimensional domain, called $\Omega^{0}$ below, see Figure 1. It can be seen as a simple model for lungs.

The domain $\Omega^{0}$ is constructed in an infinite number of steps, starting from a simple polygonal T-shaped domain of $\mathbb{R}^{2}$, see Figure 1, called $Y^{0}$ below; we call $Y^{n}$ the domain obtained at step $n$ : $Y^{n+1}$ is obtained by glueing $2^{n+1}$ dilated/translated copies of $Y^{0}$, with the dilation factor of $1 / 2^{n+1}$, to $Y^{n}$. Thus, $Y^{0} \subset Y^{1} \subset \ldots \subset \Omega^{0}$. We say that $\Omega^{0}$ is self-similar, because $\Omega^{0} \backslash \overline{Y^{n}}$ is made out of $2^{n+1}$ dilated copies of $\Omega^{0}$ with the dilation factor of $1 / 2^{n+1}$.

\footnotetext{
Keywords and phrases. Domains with fractal boundaries, Helmholtz equation, Neumann boundary conditions, transparent boundary conditions.

1 UFR Mathématiques, Université Paris 7, Case 7012, 75251 Paris Cedex 05, France and Laboratoire Jacques-Louis Lions, Université Paris 6, 75252 Paris Cedex 05, France. achdou@math.jussieu.fr

2 CNRS, UMPA, UMR 5669, 46, Allée d'Italie, 69364 Lyon Cedex 07, France. csabot@umpa.ens-lyon.fr

3 IRMAR, Université de Rennes 1, Rennes, France. nicoletta.tchou@univ-rennes1.fr
}

(C) EDP Sciences, SMAI 2006 
As we shall see below, the boundary of $\Omega^{0}$ is made up of three parts; two straight lines, the bottom (resp. top) boundary $\Gamma^{0}$ (resp. $\Gamma^{\infty}$ ) of $\Omega^{0}$, and the lateral part of the boundary $\Sigma^{0}$. Since the exchanges between the lungs and the circulatory system take place only in the last generations of the lung tree (the smallest structures), reasonable models for the diffusion of e.g. oxygen may involve non homogeneous Neumann or Robin conditions on the top boundary $\Gamma^{\infty}$. Similarly, the lungs are mechanically coupled to the diaphragm, which also implies non homogeneous boundary conditions on $\Gamma^{\infty}$, if one is interested in a coupled fluid-structure model.

The present paper is devoted to some simple elliptic boundary value problems in $\Omega^{0}$ : we discuss Poisson problems with the Laplace and Helmholtz equations. For example, the Helmholtz equation is satisfied by time harmonic acoustic waves. The Laplace equation arises in e.g. electrostatics for computing the electrical potential, or in the simplest fluid models (potential flow). The content of this paper can be applied to more involved fluid models, e.g. Stokes equations, but we decided to focus on simple equations in order to stress the general ideas. Similarly, what follows applies to the equation $\operatorname{div}(\chi \operatorname{grad} \mathrm{w})=0$, where $\chi$ is a symmetric positive definite constant tensor.

Partial differential equations in domain with fractal boundaries or fractal interfaces is a relatively new topic: variational techniques have been developed, involving new results on functional analysis, see $[12,13,19]$. A very nice theory on variational problems in fractal media is given in [18]. Some numerical simulations are described in $[24,25]$.

In this paper, we shall discuss Poisson problems with Dirichlet conditions on $\Gamma^{0}$, homogeneous Neumann conditions on $\Sigma^{0}$ and Neumann conditions on $\Gamma^{\infty}$. Since the boundary of $\Omega^{0}$ is extremely irregular, a good way to give sense to these problems is to use variational or weak formulations. For that, we need basic results on Sobolev spaces on $\Omega^{0}$, like Poincaré's inequalities, Sobolev imbeddings, extension and trace results. As we shall see below, the domain $\Omega^{0}$ is not a $(\epsilon, \delta)$ domain as defined in Jones [9] or Jonsson and Wallin [10], or equivalently in dimension two a quasi-disk, see Maz'ja [17]. Thus the classical results on Sobolev spaces see e.g. [17] cannot be applied and one must check carefully which results hold in the present case. These results will give sense to the above-mentioned non homogeneous Neumann conditions on $\Gamma^{\infty}$.

Once existence and uniqueness results are obtained, one may wonder how to compute efficiently the solutions with e.g. finite elements. In numerical simulations, it is not possible to completely represent the domain $\Omega^{0}$, for this would imply infinite memory and computing time. Therefore, we shall be interested in computing the restrictions of the solution to the domain $Y^{n}$, where $n$ is a fixed integer (one can take $n=0$ for example). Staying at the continuous level, we shall see in Algorithms 1 and 2, see Sections 5.2.2 and 6.2 below, that it is possible to find the solution in $Y^{n}$ by successively solving $1+2+\cdots+2^{n}$ boundary value problems in the elementary domain $Y^{0}$, with what we call transparent boundary conditions on the top part of the boundary of $Y^{0}$.

Transparent boundary conditions were proposed in computational physics for linear partial differential equations with constant coefficients, for which the Green functions are known, and in particular in electromagnetism, where one deals frequently with unbounded domains. They allow the solution to be computed in a bounded domain without errors. We propose to adapt the idea to the present situation. There is a huge amount of literature on transparent boundary conditions, see e.g. [11] for one of the first papers on the subject.

In this work, we discuss transparent boundary conditions for both the Laplace and Helmholtz equations, in the case when the Neumann data on $\Gamma^{\infty}$ is zero. Transparent boundary conditions for nonzero Neumann data will be discussed in [1]. The transparent conditions involve nonlocal operators, which may be called Dirichlet to Neumann operators. They will be computed, or more precisely approximated up to an arbitrary accuracy, by taking advantage of the self-similarity in the geometry. In the case of Laplace's equation, the Dirichlet to Neumann operator is approximated as the limit of an inductive sequence, see Section 5.2.3 below. In the case of the Helmholtz equation, the Dirichlet to Neumann operators (depending on the pulsation of the related harmonic wave), can be approximated by performing iterations of a renormalization operator, see Section 6.3 below. The method developed in this paper is reminiscent of some of the techniques involved in the theoretical analysis of finitely ramified fractals (see $[6,20,22,23]$ for numerical simulations). 
In this paper, we stay at the continuous level, but the methods presented below have their discrete counterpart with finite elements: the numerical methods and results for Laplace's equation are presented in [1]. Numerical results for the Helmholtz equation, and applications to the computation of the spectrum and to time-dependent problems are discussed in [2].

The paper is organized as follows: in Section 2, the geometry is presented in detail. In Section 3, we give and prove relevant theoretical results on Sobolev spaces. The boundary value problems with Laplace's equation are discussed in Section 4. In Section 5, we deal with the transparent boundary conditions, and with the computation of the nonlocal Dirichlet to Neumann operator. Section 6 is devoted to the Helmholtz equation, and to the use of transparent boundary conditions for computing the solution in a truncated domain $Y^{n}$. For the reader's ease, some of the proofs are given in separate sections at the end of the paper.

Finally, let us stress that most of the results and methods described below can be used for other geometries: for example, except for what concerns nonhomogeneous Neumann conditions on $\Gamma^{\infty}$, the whole content of the paper can be adapted to the famous case of the Koch flake.

\section{The GeOMETRY OF THE MODEL PROBLEM}

\subsection{The domain $\Omega^{0}$}

Consider the following T-shaped subset of $\mathbb{R}^{2}$

$$
Y^{0}=\operatorname{Interior}(([-1,1] \times[0,2]) \cup([-2,2] \times[2,3])) .
$$

Let $F_{1}$ and $F_{2}$ be the affine maps in $\mathbb{R}^{2}$

$$
F_{1}(x)=\left(-\frac{3}{2}+\frac{x_{1}}{2}, 3+\frac{x_{2}}{2}\right), \quad F_{2}(x)=\left(\frac{3}{2}+\frac{x_{1}}{2}, 3+\frac{x_{2}}{2}\right) .
$$

Note that $F_{1}$ is the homothety of ratio $\frac{1}{2}$ and center $(-3,6)$, and $F_{2}$ is the homothety of ratio $\frac{1}{2}$ and center $(3,6)$.

For any integer $n \geq 1$, we call $\mathcal{A}_{n}$ the set containing all the maps from $\{1, \ldots, n\}$ to $\{1,2\}$, (note that the cardinality of $\mathcal{A}_{n}$ is $2^{n}$ ) and for $\sigma \in \mathcal{A}_{n}$, we define the affine map in $\mathbb{R}^{2}$

$$
\mathcal{M}_{\sigma}\left(F_{1}, F_{2}\right)=F_{\sigma(1)} \circ \cdots \circ F_{\sigma(n)}
$$

Let us agree that $\mathcal{A}_{0}=\{0\}$ and that $\mathcal{M}_{0}\left(F_{1}, F_{2}\right)$ is the identity. The open domain $\Omega^{0}$ is constructed as an infinite union of subsets of $\mathbb{R}^{2}$ obtained by translating/dilating $Y^{0}$ :

$$
\Omega^{0}=\operatorname{Interior}\left(\cup_{n=0}^{\infty} \cup_{\sigma \in \mathcal{A}_{n}} \mathcal{M}_{\sigma}\left(F_{1}, F_{2}\right)\left(\overline{Y^{0}}\right)\right)
$$

The construction of $\Omega^{0}$ is displayed in Figure 1 . One can see that $\Omega^{0} \subset(-3,3) \times(0,6)$.

Remark 1. Note that similar construction may be done, using dilations with ratii $\alpha^{n}, n \in \mathbb{N}$, with $\alpha \in(0,1 / 2]$; here we have chosen $\alpha=1 / 2$. 

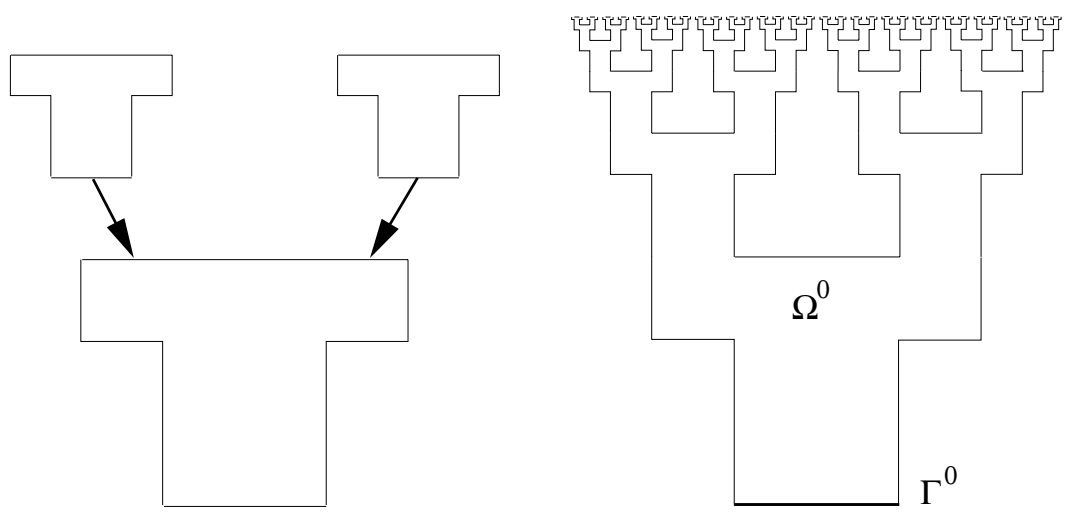

FIGURE 1. Left: the first step of construction: two dilated/translated copies of $Y^{0}$ are attached to $Y^{0}$. Right: the ramified domain $\Omega^{0}$ (only a few generations are displayed).

\subsection{The domain $\Omega^{0}$ is not a $(\epsilon, \delta)$ domain}

For completeness, we recall the definition of a $(\epsilon, \delta)$ domain, (see Jones [9], and Jonsson and Wallin [10]): an open connected subset $\Omega$ of $\mathbb{R}^{d}$ is a $(\epsilon, \delta)$ domain, if whenever $x, y \in \Omega$ and $|x-y|<\delta$, there exists a rectifiable arc $\gamma \subset \Omega$ with length $\ell(\gamma)$ joining $x$ and $y$ such that:

1. $\ell(\gamma) \leq|x-y| / \epsilon$

2. using the notation $d(z)=\inf _{t \notin \Omega}|z-t|$ for $z \in \Omega$, we have $d(z) \geq(\epsilon|x-z||y-z|) /|x-y|$ for all $z \in \gamma$. In dimension two, the notion of $(\epsilon, \delta)$ domain is equivalent to that of quasi-disk, see Maz'ja [17].

It is important to observe that $\Omega^{0}$ is not a $(\epsilon, \delta)$ domain: indeed, take the two points $A=(-3 / 2,5 / 2)$ and $B=(3 / 2,5 / 2)$, and call $A_{n}=\left(F_{1} \circ F_{2}^{n}\right)(B), B_{n}=\left(F_{2} \circ F_{1}^{n}\right)(A)$, we have that

- $\lim _{n \rightarrow \infty} A_{n}=\lim _{n \rightarrow \infty} B_{n}=(0,6)$, therefore $\lim _{n \rightarrow \infty}\left|A_{n} B_{n}\right|=0$;

- $A_{n} \in \Omega^{0}$ and $B_{n} \in \Omega^{0}$;

- the length of any curve joining $A_{n}$ and $B_{n}$ that is contained in $\Omega^{0}$ is greater than 3 .

Therefore, when studying the Sobolev spaces in $\Omega^{0}$, we shall not be able to use the theory of Jones [9]. In particular, general extension results do not apply to $\Omega^{0}$.

\subsection{The boundary of $\Omega^{0}$}

We define the bottom boundary of $\Omega^{0}$ by $\Gamma^{0}=([-1,1] \times\{0\})$ and $\Sigma^{0}=\partial \Omega^{0} \cap\left\{\left(x_{1}, x_{2}\right) ; x_{1} \in \mathbb{R}, 0<x_{2}<6\right\}$. Calling $\Gamma^{\infty}=[-3,3] \times\{6\}$, one can verify that

$$
\partial \Omega^{0}=\Gamma^{0} \cup \Sigma^{0} \cup \Gamma^{\infty}
$$

and that the sets in the right hand side of (4) are disjoint.

Remark 2. Note that

1. $\Gamma^{\infty}$ is the unique compact set which is invariant with respect to the set of contraction maps $\left\{F_{1}, F_{2}\right\}$, i.e. such that $\Gamma^{\infty}=F_{1}\left(\Gamma^{\infty}\right) \cup F_{2}\left(\Gamma^{\infty}\right)$, see $[8,14]$. The similarity dimension of $\Gamma^{\infty}$ is one, see [14] for the definition.

2. The Hausdorff dimension of $\partial \Omega^{0}$ is one.

The second point will not be used for what follows (this is why its proof is omitted). 


\subsection{Various subdomains of $\Omega^{0}$}

For what follows, it is important to define the polygonal open domain obtained by stopping the above construction at the step $N, N \geq 0$.

$$
Y^{N}=\operatorname{Interior}\left(\cup_{n=0}^{N} \cup_{\sigma \in \mathcal{A}_{n}} \mathcal{M}_{\sigma}\left(F_{1}, F_{2}\right)\left(\overline{Y^{0}}\right)\right) .
$$

It will be useful to define the ramified set $\Omega^{N}$

$$
\Omega^{N}=\Omega^{0} \backslash \overline{Y^{N-1}}=\text { Interior }\left(\cup_{n=N}^{\infty} \cup_{\sigma \in \mathcal{A}_{n}} \mathcal{M}_{\sigma}\left(F_{1}, F_{2}\right)\left(\overline{Y^{0}}\right)\right) .
$$

The following self-similarity property is true: $\Omega^{N}$ is the union of $2^{N}$ nonoverlapping translated copies of $\frac{1}{2^{N}} \cdot \Omega^{0}$, i.e.

where

$$
\Omega^{N}=\cup_{\sigma \in \mathcal{A}_{N}} \Omega^{\sigma}
$$

$\Omega^{\sigma}=\mathcal{M}_{\sigma}\left(F_{1}, F_{2}\right)\left(\Omega^{0}\right)$.

The bottom boundary of $\Omega^{N}$ is defined by

$$
\Gamma^{N}=\cup_{\sigma \in \mathcal{A}_{N}} \Gamma^{\sigma} \subset\left\{x: x_{2}=3 \sum_{i=0}^{N-1} 2^{-i}\right\}
$$

where

$$
\Gamma^{\sigma}=\mathcal{M}_{\sigma}\left(F_{1}, F_{2}\right)\left(\Gamma^{0}\right)
$$

Let us stress that $\Gamma^{N}$ is strictly contained in $\partial Y^{N-1} \cap\left\{x: x_{2}=3 \sum_{i=0}^{N-1} 2^{-i}\right\}$.

\section{Some FUnCtion SPACES}

We aim at studying some elliptic boundary value problems in $\Omega^{0}$ and their weak formulation. For that, we need to first introduce some natural Sobolev spaces on $\Omega^{0}$, and present some of their properties which we did not find in the literature.

Let $q$ be a real number such that $q \geq 1$. For a nonnegative integer $n$ and a real number $s$, we define the Sobolev space $W^{s, q}\left(Y^{n}\right)$ as in [3,4] (note that $Y^{n}$ has a Lipschitz regular boundary, so a full theory is available for the spaces $\left.W^{s, q}\left(Y^{n}\right)\right)$.

For $n \geq 0$ and $\sigma \in \mathcal{A}_{n}$, we shall also consider the function space $W^{1, q}\left(\Omega^{\sigma}\right)=\left\{v \in L^{q}\left(\Omega^{\sigma}\right)\right.$ s.t. $\left.\nabla v \in\left(L^{q}\left(\Omega^{\sigma}\right)\right)^{2}\right\}$. Similarly, for all positive integers $p$, it is possible to define $W^{p, q}\left(\Omega^{\sigma}\right)$ as the space of functions whose partial derivatives up to order $p$ belong to $L^{q}\left(\Omega^{\sigma}\right)$. Since $\Omega^{\sigma}$ is not a quasi-disk, the extension and trace results in $[9,10,17]$ cannot be applied.

Of course, for all $n, n^{\prime}, 0 \leq n \leq n^{\prime}, \sigma \in \mathcal{A}_{n}, \eta \in \mathcal{A}_{n^{\prime}}$ such that $\Gamma^{\eta} \subset \overline{\Omega^{\sigma}}$, it is possible to define the trace of $v \in W^{1, q}\left(\Omega^{\sigma}\right)$ on $\Gamma^{\eta}$. The trace operator on $\Gamma^{\eta}$ is bounded from $W^{1, q}\left(\Omega^{\sigma}\right)$ to $L^{q}\left(\Gamma^{\eta}\right)$, and one can define the closed subspace of $W^{1, q}\left(\Omega^{\sigma}\right)$ :

$$
\mathcal{V}^{q}\left(\Omega^{\sigma}\right)=\left\{v \in W^{1, q}\left(\Omega^{\sigma}\right) \text { s.t. }\left.v\right|_{\Gamma^{\sigma}}=0\right\} .
$$

In the case $q=2$, the spaces are Hilbert spaces, and we use the special notation $H^{p}\left(\Omega^{0}\right)=W^{p, 2}\left(\Omega^{0}\right)$ and $\mathcal{V}\left(\Omega^{\sigma}\right)=\mathcal{V}^{2}\left(\Omega^{\sigma}\right)$.

Finally, we shall also use the Cartesian product spaces $W^{p, q}\left(\Omega^{n}\right)=\prod_{\sigma \in \mathcal{A}_{n}} W^{p, q}\left(\Omega^{\sigma}\right)$, with the norm $\|v\|_{W^{p, q}\left(\Omega^{n}\right)}=\left(\sum_{\sigma \in \mathcal{A}_{n}}\left\|\left.v\right|_{\Omega^{\sigma}}\right\|_{W^{p, q}\left(\Omega^{\sigma}\right)}^{q}\right)^{1 / q}$. The restriction of $v \in W^{p, q}\left(\Omega^{0}\right)$ to $\Omega^{n}$ belongs to $W^{p, q}\left(\Omega^{n}\right)$ and its trace on $\Gamma^{n}$ belongs to $L^{q}\left(\Gamma^{n}\right)=\prod_{\sigma \in \mathcal{A}_{n}} L^{q}\left(\Gamma^{\sigma}\right)$.

In what follows, for a function $u$ integrable on $\Gamma^{\sigma}, \sigma \in \mathcal{A}_{n}, n \geq 0$, the notation $\langle u\rangle_{\Gamma^{\sigma}}$ will be used for the mean value of $u$ on $\Gamma^{\sigma}$. 

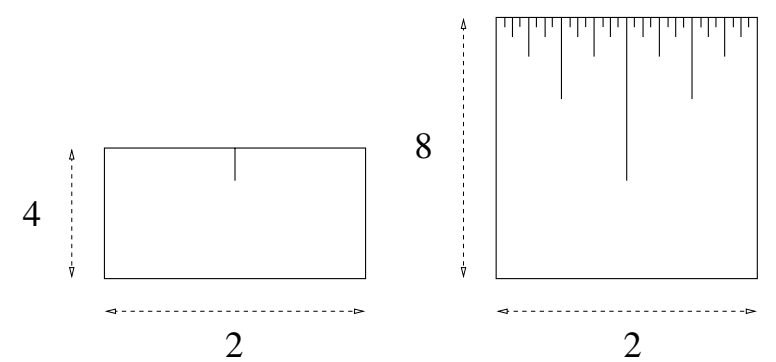

FiguRE 2. Left: the set $\widehat{\mathcal{Q}^{0}}$. Right: the open set $\omega^{0}$ (only the longest fractures are displayed).

We will also use the notation $\lesssim$ to indicate that there may arise constants in the estimates, which are independent of the index $n$ in $\Omega^{n}$ or $Y^{n}$, or the index $\sigma$ in $\Omega^{\sigma}$.

\subsection{Poincaré's inequality and consequences}

Theorem 1 (Poincaré's inequality). For any $u \in \mathcal{V}^{q}\left(\Omega^{0}\right)$,

$$
\|u\|_{L^{q}\left(\Omega^{0}\right)} \leq 8 q^{-\frac{1}{q}}\|\nabla u\|_{L^{q}\left(\Omega^{0}\right)} .
$$

Proof. The proof is done by explicitly constructing a measure preserving and one to one mapping from $\Omega^{0}$ onto the set $\omega^{0}$ displayed in Figure 2. The set $\omega^{0}$ is obtained by removing an infinite number of vertical segments from the rectangle $(-1,1) \times(0,8)$. It can be constructed by assembling translated/dilated copies of the set $\widehat{\mathcal{Q}^{0}}$ displayed on the left of Figure 2. For the reader's ease, the details of the proof are postponed to Section 8.

Corollary 1. There exists a positive constant $\mathrm{C}$ such that for all $u \in W^{1, q}\left(\Omega^{0}\right)$,

$$
\|u\|_{L^{q}\left(\Omega^{0}\right)}^{q} \leq \mathrm{C}\left(\|\nabla u\|_{L^{q}\left(\Omega^{0}\right)}^{q}+\left\|\left.u\right|_{\Gamma^{0}}\right\|_{L^{q}\left(\Gamma^{0}\right)}^{q}\right) .
$$

Proof. Define $W^{1-\frac{1}{q}, q}\left(\Gamma^{0}\right)$ as the space of the traces on $\Gamma^{0}$ of the functions belonging to $W^{1, q}\left(Y^{0}\right)$, endowed with the norm

$$
\|u\|_{W^{1-\frac{1}{q}, q}\left(\Gamma^{0}\right)}=\inf _{v \in W^{1, q}\left(Y^{0}\right),\left.v\right|_{\Gamma^{0}}=u}\|v\|_{W^{1, q}\left(Y^{0}\right)} .
$$

It is a classical result that for all $v \in W^{1, q}\left(Y^{0}\right)$,

$$
\left\|\left.v\right|_{\Gamma^{0}}\right\|_{W^{1-\frac{1}{q}, q}\left(\Gamma^{0}\right)} \lesssim\left(\|\nabla v\|_{L^{q}\left(Y^{0}\right)}^{q}+\left\|\left.v\right|_{\Gamma^{0}}\right\|_{L^{q}\left(\Gamma^{0}\right)}^{q}\right)^{\frac{1}{q}} .
$$

Moreover there exists a continuous lifting operator $L: W^{1-\frac{1}{q}, q}\left(\Gamma^{0}\right)$ to $W^{1, q}\left(Y^{0}\right)$ such that $\forall v \in W^{1-\frac{1}{q}, q}\left(\Gamma^{0}\right)$, $\left.(L v)\right|_{\Gamma^{1}}=0$, and

$$
\|L v\|_{W^{1, q}\left(Y^{0}\right)} \lesssim\|v\|_{W^{1-\frac{1}{q}, q}\left(\Gamma^{0}\right)} .
$$

For $u \in W^{1, q}\left(\Omega^{0}\right)$, calling $H(u)$ the function defined in $\Omega^{0}$ by extending $L\left(\left.u\right|_{\Gamma^{0}}\right)$ by 0 outside $Y^{0}$, we have that $u-H(u) \in \mathcal{V}^{q}\left(\Omega^{0}\right)$, and from (12), (15) and (16),

$$
\begin{aligned}
\|u-H(u)\|_{L^{q}\left(\Omega^{0}\right)} & \leq 8 q^{-\frac{1}{q}}\|\nabla(u-H(u))\|_{L^{q}\left(\Omega^{0}\right)} \leq 8 q^{-\frac{1}{q}}\left(\|\nabla u\|_{L^{q}\left(\Omega^{0}\right)}+\|\nabla(H(u))\|_{L^{q}\left(\Omega^{0}\right)}\right) \\
& \lesssim\|\nabla u\|_{L^{q}\left(\Omega^{0}\right)}+\left\|\left.u\right|_{\Gamma^{0}}\right\|_{W^{1-\frac{1}{q}, q}\left(\Gamma^{0}\right)} \\
& \lesssim\|\nabla u\|_{L^{q}\left(\Omega^{0}\right)}+\left\|\left.u\right|_{\Gamma^{0}}\right\|_{L^{q}\left(\Gamma^{0}\right)} .
\end{aligned}
$$

We obtain (13) from the previous inequality, using (15) and (16) again. 
Remark 3. Results similar to Corollary 1 can be proved, for instance: there exists a positive constant $\mathrm{C}$ such that for all $u \in W^{1, q}\left(\Omega^{0}\right)$,

$$
\|u\|_{L^{q}\left(\Omega^{0}\right)}^{q} \leq \mathrm{C}\left(\|\nabla u\|_{L^{q}\left(\Omega^{0}\right)}^{q}+\left|\langle u\rangle_{\Gamma^{0}}\right|^{q}\right) .
$$

By a simple scaling argument, we obtain from (13) the

Corollary 2. There exists a positive constant $\mathrm{C}$ such that for all integer $n \geq 0$, and for all $\sigma \in \mathcal{A}_{n}$, for all $u \in W^{1, q}\left(\Omega^{\sigma}\right)$, ( $\Omega^{\sigma}$ is defined in (8))

$$
\|u\|_{L^{q}\left(\Omega^{\sigma}\right)}^{q} \leq \mathrm{C}\left(2^{-n q}\|\nabla u\|_{L^{q}\left(\Omega^{\sigma}\right)}^{q}+2^{-n}\left\|\left.u\right|_{\Gamma^{\sigma}}\right\|_{L^{q}\left(\Gamma^{\sigma}\right)}^{q}\right),
$$

where $\Gamma^{\sigma}$ is defined by (10), and for all $u \in W^{1, q}\left(\Omega^{n}\right)$

$$
\|u\|_{L^{q}\left(\Omega^{n}\right)}^{q} \leq \mathrm{C}\left(2^{-n q}\|\nabla u\|_{L^{q}\left(\Omega^{n}\right)}^{q}+2^{-n}\left\|\left.u\right|_{\Gamma^{n}}\right\|_{L^{q}\left(\Gamma^{n}\right)}^{q}\right) .
$$

Lemma 1. There exists a positive constant $\mathrm{C}$ such that for all $u \in W^{1, q}\left(\Omega^{0}\right)$, for all $N \geq 0$,

$$
\|u\|_{L^{q}\left(\Omega^{N}\right)}^{q} \leq \mathrm{C} 2^{-N}\left(\|\nabla u\|_{L^{q}\left(\Omega^{0}\right)}^{q}+\left\|\left.u\right|_{\Gamma^{0}}\right\|_{L^{q}\left(\Gamma^{0}\right)}^{q}\right) .
$$

Proof. For $\sigma \in \mathcal{A}_{n}$, we use a trace inequality on $\mathcal{M}_{\sigma}\left(F_{1}, F_{2}\right)\left(Y^{0}\right)$ : for a constant $\mathrm{C}$ independent on $n$, we have

$$
2^{n}\left\|\left.u\right|_{\mathcal{M}_{\sigma}\left(F_{1}, F_{2}\right)\left(\Gamma^{1}\right)}\right\|_{L^{q}\left(\mathcal{M}_{\sigma}\left(F_{1}, F_{2}\right)\left(\Gamma^{1}\right)\right)}^{q} \leq \mathrm{C}\|\nabla u\|_{L^{q}\left(\mathcal{M}_{\sigma}\left(F_{1}, F_{2}\right)\left(Y^{0}\right)\right)}^{q}+2^{n}\left\|\left.u\right|_{\Gamma^{\sigma}}\right\|_{L^{q}\left(\Gamma^{\sigma}\right)}^{q},
$$

because $\Gamma^{1}$ and $\Gamma^{0}$ (resp. $\mathcal{M}_{\sigma}\left(F_{1}, F_{2}\right)\left(\Gamma^{1}\right)$ and $\left.\Gamma^{\sigma}=\mathcal{M}_{\sigma}\left(F_{1}, F_{2}\right)\left(\Gamma^{0}\right)\right)$ have the same measures. Summing (21) over $\sigma \in \mathcal{A}_{n}$, we obtain that

$$
2^{n}\left\|\left.u\right|_{\Gamma^{n+1}}\right\|_{L^{q}\left(\Gamma^{n+1}\right)}^{q} \leq \mathrm{C} \sum_{\sigma \in \mathcal{A}_{n}}\|\nabla u\|_{L^{q}\left(\mathcal{M}_{\sigma}\left(F_{1}, F_{2}\right)\left(Y^{0}\right)\right)}^{q}+2^{n}\left\|\left.u\right|_{\Gamma^{n}}\right\|_{L^{q}\left(\Gamma^{n}\right)}^{q} .
$$

Multiplying (22) by $2^{-n}$ and summing up from $n=0$ to $N-1$, we obtain that

$$
\left\|\left.u\right|_{\Gamma^{N}}\right\|_{L^{q}\left(\Gamma^{N}\right)}^{q} \leq \mathrm{C}\left(\|\nabla u\|_{L^{q}\left(Y^{N-1}\right)}^{q}+\left\|\left.u\right|_{\Gamma^{0}}\right\|_{L^{q}\left(\Gamma^{0}\right)}^{q}\right) .
$$

Substituting this into (19), we obtain (20).

Theorem 2 (compactness). The imbedding of $W^{1, q}\left(\Omega^{0}\right)$ in $L^{q}\left(\Omega^{0}\right)$ is compact.

Proof. From (20), we have $\left\|u-1_{Y^{N}} u\right\|_{L^{q}\left(\Omega^{0}\right)} \leq \mathrm{C} 2^{-\frac{N}{q}}\|u\|_{W^{1, q}\left(\Omega^{0}\right)}$. On the other hand, the imbedding of $W^{1, q}\left(Y^{N}\right)$ in $L^{q}\left(Y^{N}\right)$ is compact. Combining the previous two remarks yields the desired result.

\subsection{Extensions and traces}

\subsubsection{The main extension result}

We aim at constructing an extension operator mapping a function defined on $\Omega^{0}$ to a function defined in a simple polygonal domain $\widehat{\Omega}^{0}$. We choose $\widehat{\Omega}^{0}$ as the trapezoidal domain with vertices $\left(-\frac{3}{2}, 0\right),\left(\frac{3}{2}, 0\right),(-3,6)$, and $(3,6)$. Note that $\Omega^{0} \subset \widehat{\Omega}^{0}$. 
We know that there does not exist $\epsilon>0$ and $\delta>0$ such that $\Omega^{0}$ is a $(\epsilon, \delta)$ domain (see $[9,10,17]$ ). Therefore, from [9], (Thms. 1 and 3), there must exist an integer $p$ and a real number $q, 1 \leq q \leq \infty$, such that there does not exist a bounded extension operator from $W^{p, q}\left(\Omega^{0}\right)$ to $W^{p, q}\left(\widehat{\Omega}^{0}\right)$. In fact, it is very easy to see that there is no bounded extension operator from $H^{1}\left(\Omega^{0}\right)$ to $H^{1}\left(\widehat{\Omega}^{0}\right)$ : indeed it is possible to construct a function $u \in H^{1}\left(\Omega^{0}\right)$ such that

$$
\begin{aligned}
& u(x)=1, \quad x \in F_{1}\left(\Omega^{0}\right), \\
& u(x)=0, \quad x \in F_{2}\left(\Omega^{0}\right) .
\end{aligned}
$$

Note that $\Delta$, the open segment of the straight line joining the points $(0,6)$ and $\left(-\frac{7}{4}, 3\right)$, is contained in $\Omega^{0}$, (indeed the whole triangle $\left\{x: 2 x_{1}-x_{2}<-6,3 x_{1}-2 x_{2}>-12,3<x_{2}\right\}$ is contained in $\Omega^{0}$ ). Similarly, $\Delta^{\prime}$, the open segment of the straight line joining the points $(0,6)$ and $\left(\frac{7}{4}, 3\right)$, is contained in $\Omega^{0}$ (the whole triangle $\left\{x:-2 x_{1}-x_{2}<-6,-3 x_{1}-2 x_{2}>-12,3<x_{2}\right\}$ is contained in $\left.\Omega^{0}\right)$. It is clear that $\left.u\right|_{\Delta}=1$ and that $\left.u\right|_{\Delta^{\prime}}=0$. If there existed a bounded extension $\mathcal{J}$ from $H^{1}\left(\Omega^{0}\right)$ to $H^{1}\left(\widehat{\Omega}^{0}\right)$, then we would have $\left.\mathcal{J}(u)\right|_{\Delta}=1$ and $\left.\mathcal{J}(u)\right|_{\Delta^{\prime}}=0$, in contradiction with the fact that $\mathcal{J}(u) \in H^{1}\left(\widehat{\Omega}^{0}\right)$.

The previous observation indicates that the following extension result is in some sense optimal:

Theorem 3. There exists an extension operator $\mathcal{J}$ bounded from $W^{1, q}\left(\Omega^{0}\right)$ to $W^{1, q}\left(\widehat{\Omega}^{0}\right)$, for all $q, 1 \leq q<2$.

Proof. Since the proof of Theorem 3 is rather long, we postpone it to Section 9.

Remark 4. Of course, since $\Omega^{0}$ is a bounded domain, the extension operator $\mathcal{J}$ is bounded from $H^{1}\left(\Omega^{0}\right)$ to $W^{1, q}\left(\hat{\Omega}^{0}\right)$, for $q, 1 \leq q<2$.

As a consequence of Theorem 3, we have the Sobolev imbeddings:

Proposition 1 (Sobolev imbeddings). Let $q$ be a real number such that $1 \leq q<2$, we have the continuous imbeddings:

$$
W^{1, q}\left(\Omega^{0}\right) \subset L^{p}\left(\Omega^{0}\right), \quad \forall p, \quad 1 \leq p \leq q^{*}, \quad q^{*}=\frac{2 q}{2-q},
$$

and the imbedding is compact if $p<q^{*}$.

Furthermore, for all $q, p, 1 \leq q<2,1 \leq p \leq q^{*}$, there exists a constant $\mathrm{C}$ such that for all $N \geq 0$, $u \in W^{1, q}\left(\Omega^{N}\right)$,

$$
\|u\|_{L^{p}\left(\Omega^{N}\right)}^{p} \leq \mathrm{C}\left(2^{\frac{2 N(p-q)-q p N}{q}}\|\nabla u\|_{L^{q}\left(\Omega^{N}\right)}^{p}+2^{\frac{N(p-2 q)}{q}}\|u\|_{L^{q}\left(\Gamma^{N}\right)}^{p}\right) .
$$

For all real number $p, 1 \leq p<\infty, H^{1}\left(\Omega^{0}\right) \subset L^{p}\left(\Omega^{0}\right)$ with continuous and compact imbedding.

\subsubsection{Density results}

We denote with $\mathcal{C}^{\infty}\left(\overline{\Omega^{0}}\right)$ (resp. $\mathcal{C}^{\infty}\left(\overline{\Omega^{0}}\right)$ ) the space of the restrictions to $\Omega^{0}$ (resp. $\widehat{\Omega}^{0}$ ) of the functions in $\mathcal{C}^{\infty}\left(\mathbb{R}^{2}\right)$. We call $\mathcal{R}: \mathcal{C}^{\infty}\left(\overline{\widehat{\Omega}^{0}}\right) \rightarrow \mathcal{C}^{\infty}\left(\overline{\Omega^{0}}\right)$ the operator which maps $v \in \mathcal{C}^{\infty}\left(\overline{\widehat{\Omega}^{0}}\right)$ to its restriction on $\Omega^{0}$.

Theorem 4. For $q, 1 \leq q<2$, there exist a constant c and a sequence of linear operators $\left(\mathcal{S}_{n}\right)_{n \in \mathbb{N}}$ from $W^{1, q}\left(\Omega^{0}\right)$ to $\mathcal{C}^{\infty}\left(\overline{\Omega^{0}}\right)$ such that

$$
\forall u \in W^{1, q}\left(\Omega^{0}\right), \quad\left\|\left(\mathcal{R} \circ \mathcal{S}_{n}\right)(u)\right\|_{W^{1, q}\left(\Omega^{0}\right)} \leq\left\|\mathcal{S}_{n} u\right\|_{W^{1, q}\left(\widehat{\Omega}^{0}\right)} \leq \mathrm{c}\|u\|_{W^{1, q}\left(\Omega^{0}\right)},
$$

and

$$
\forall u \in W^{1, q}\left(\Omega^{0}\right), \quad \lim _{n \rightarrow \infty}\left\|u-\left(\mathcal{R} \circ \mathcal{S}_{n}\right)(u)\right\|_{W^{1, q}\left(\Omega^{0}\right)}=0 .
$$

Therefore, $\mathcal{C}^{\infty}\left(\overline{\Omega^{0}}\right)$ is dense in $W^{1, q}\left(\Omega^{0}\right)$. 
Proof. We know that for all $q, 1 \leq q<2$, (in fact, this is true for all $q, 1 \leq q<\infty$ ), $\mathcal{C}^{\infty}\left(\overline{\widehat{\Omega}^{0}}\right)$ is dense in $W^{1, q}\left(\widehat{\Omega}^{0}\right)$ and that there exists a sequence of linear operators $\left(\widehat{\mathcal{S}}_{n}\right)_{n \in \mathbb{N}}$ from $W^{1, q}\left(\widehat{\Omega}^{0}\right)$ to $\mathcal{C} \infty\left(\overline{\widehat{\Omega}^{0}}\right)$ such that

$$
\forall v \in W^{1, q}\left(\widehat{\Omega}^{0}\right), \quad \lim _{n \rightarrow \infty}\left\|v-\widehat{\mathcal{S}}_{n} v\right\|_{W^{1, q}\left(\widehat{\Omega}^{0}\right)}=0,
$$

and for a constant $\mathrm{c}$,

$$
\forall v \in W^{1, q}\left(\widehat{\Omega}^{0}\right), \quad\left\|\widehat{\mathcal{S}}_{n} v\right\|_{W^{1, q}\left(\widehat{\Omega}^{0}\right)} \leq \mathrm{c}\|v\|_{W^{1, q}\left(\widehat{\Omega}^{0}\right)} .
$$

Consider an extension operator $\mathcal{J}$ as in Theorem 3. The operators $\mathcal{S}_{n}=\widehat{\mathcal{S}}_{n} \circ \mathcal{J}$ answer the question.

\subsubsection{Traces}

Recall that if $u \in \mathcal{C}^{\infty}\left(\overline{\widehat{\Omega}^{0}}\right)$, then the trace of $\mathcal{R}(u)$ on $\Gamma^{\infty}$ coincides with the trace of $u$ on $\Gamma^{\infty}$.

Take $q, 1<q$ and call $\mathcal{N}_{m}^{q}$ the mapping

$$
\mathcal{N}_{m}^{q}: W^{1, q}\left(\Omega^{0}\right) \rightarrow \mathbb{R}_{+}, \quad \mathcal{N}_{m}^{q}(v)=\left\|\left.v\right|_{\Gamma^{m}}\right\|_{L^{q}\left(\Gamma^{m}\right)}^{q} .
$$

It is easy to check the following estimate: for all integers $n, m, n>m \geq 0$ and for all $v \in W^{1, q}\left(\Omega^{0}\right)$,

$$
\left|\left\|\left.v\right|_{\Gamma^{m+1}}\right\|_{L^{q}\left(\Gamma^{m+1}\right)}^{q}-\left\|\left.v\right|_{\Gamma^{n+1}}\right\|_{L^{q}\left(\Gamma^{n+1}\right)}^{q}\right| \leq \mathrm{C} 2^{(1-q) m}\|\nabla v\|_{L^{q}\left(Y^{n} \backslash Y^{m}\right)}^{q} .
$$

Estimate (27) shows that for any $v \in W^{1, q}\left(\Omega^{0}\right),\left(\mathcal{N}_{m}^{q}(v)\right)_{m \in \mathbb{N}}$ is a Cauchy sequence in $\mathbb{R}_{+}$and that it converges to a real number $\mathcal{N}_{\infty}^{q}(v)$ as $m$ tends to infinity. It is an easy matter to prove the following

Lemma 2. Let $q$ be a real number such that $1<q$. The mapping $v \mapsto \mathcal{N}_{\infty}^{q}(v)$ is homogeneous of degree $q$. There exists a constant $\mathrm{C}$ such that for all $v \in W^{1, q}\left(\Omega^{0}\right)$,

$$
\mathcal{N}_{\infty}^{q}(v) \leq \mathrm{C}\|v\|_{W^{1, q}\left(\Omega^{0}\right)}^{q} .
$$

For $v \in \mathcal{C}^{\infty}\left(\overline{\Omega^{0}}\right)$,

$$
\mathcal{N}_{\infty}^{q}(v)=\frac{1}{3}\left\|\left.v\right|_{\Gamma^{\infty}}\right\|_{L^{q}\left(\Gamma^{\infty}\right)}^{q} .
$$

Theorem 5. Let $q$ be a real number such that $1<q<2$. The trace operator on $\Gamma^{\infty}$ defined on $\mathcal{C}^{\infty}\left(\overline{\Omega^{0}}\right)$ can be extended to a unique continuous operator from $W^{1, q}\left(\Omega^{0}\right)$ onto $W^{1-\frac{1}{q}, q}\left(\Gamma^{\infty}\right)$.

Proof. Consider a sequence of operators $\left(\mathcal{S}_{n}\right)_{n \in \mathbb{N}}$ as in Theorem 4. Let $u$ be a function in $W^{1, q}\left(\Omega^{0}\right)$. The function $\mathcal{S}_{n}(u)$ has a trace on $\Gamma^{\infty}$, belonging to $W^{1-\frac{1}{q}, q}\left(\Gamma^{\infty}\right)$, and we have from (24) that for a constant c (independent of $n$ )

$$
\left\|\left.\left(\mathcal{S}_{n}(u)\right)\right|_{\Gamma^{\infty}}\right\|_{W^{1-\frac{1}{q}, q}\left(\Gamma^{\infty}\right)} \leq c\|u\|_{W^{1, q}\left(\Omega^{0}\right)} .
$$

We recall that $\left.\left(\mathcal{R} \circ \mathcal{S}_{n}\right)(u)\right|_{\Gamma \infty}=\left.\left(\mathcal{S}_{n}(u)\right)\right|_{\Gamma \infty}$, so the same estimate holds with $\left.\left(\mathcal{R} \circ \mathcal{S}_{n}\right)(u)\right|_{\Gamma \infty}$ on the left hand side.

Therefore, one can extract a subsequence $\mathcal{S}_{\phi(n)}$ such that $\left.\mathcal{S}_{\phi(n)}(u)\right|_{\Gamma^{\infty}}$ converges weakly in $W^{1-\frac{1}{q}, q}\left(\Gamma^{\infty}\right)$ and strongly in $L^{q}\left(\Gamma^{\infty}\right)$ to some function $w \in W^{1-\frac{1}{q}, q}\left(\Gamma^{\infty}\right)$. There remains to prove that $w$ is unique (i.e. the whole sequence converges), and that $w$ depends only on $u$ and not on the sequence $\mathcal{S}_{n}$.

Assume that there exist two subsequences $\left(\mathcal{S}_{\phi(n)}(u)\right)_{n \in \mathbb{N}}$ and $\left(\mathcal{S}_{\psi(n)}(u)\right)_{n \in \mathbb{N}}$ whose traces on $\Gamma^{\infty}$ converge respectively to $w$ and $w^{\prime}$ weakly in $W^{1-\frac{1}{q}, q}\left(\Gamma^{\infty}\right)$ and strongly in $L^{q}\left(\Gamma^{\infty}\right)$. We have from (29) that

$$
\begin{aligned}
\left\|w-w^{\prime}\right\|_{L^{q}\left(\Gamma^{\infty}\right)}^{q} & =\lim _{n \rightarrow \infty}\left\|\left.\left(\left(\mathcal{R} \circ \mathcal{S}_{\phi(n)}\right)(u)\right)\right|_{\Gamma^{\infty}}-\left.\left(\left(\mathcal{R} \circ \mathcal{S}_{\psi(n)}\right)(u)\right)\right|_{\Gamma^{\infty}}\right\|_{L^{q}\left(\Gamma^{\infty}\right)}^{q} \\
& =3 \lim _{n \rightarrow \infty} \mathcal{N}_{\infty}^{q}\left(\left(\mathcal{R} \circ \mathcal{S}_{\phi(n)}\right)(u)-\left(\mathcal{R} \circ \mathcal{S}_{\psi(n)}\right)(u)\right) .
\end{aligned}
$$


From (28),

$$
\left\|w-w^{\prime}\right\|_{L^{q}\left(\Gamma^{\infty}\right)}^{q} \leq 3 C \limsup _{n \rightarrow \infty}\left\|\left(\mathcal{R} \circ \mathcal{S}_{\phi(n)}\right)(u)-\left(\mathcal{R} \circ \mathcal{S}_{\psi(n)}\right)(u)\right\|_{W^{1, q}\left(\Omega^{0}\right)}^{q},
$$

and we conclude from (25) that

$$
\left\|w-w^{\prime}\right\|_{L^{q}\left(\Gamma^{\infty}\right)}^{q}=0
$$

and therefore $w=w^{\prime}$.

The same argument leads to the fact that $w$ does not depend on the sequence of operators $\left(\mathcal{S}_{n}\right)_{n \in \mathbb{N}}$, and that if $u \in \mathcal{C}^{\infty}\left(\overline{\Omega^{0}}\right)$, then $w=\left.u\right|_{\Gamma^{\infty}}$. It can also be proved by density that if $u=\left.\hat{u}\right|_{\Omega^{0}}, \hat{u} \in W^{1, q}\left(\widehat{\Omega}^{0}\right)$, then $w$ coincides with the usual trace of $\hat{u}$ on $\Gamma^{\infty}$.

Therefore, we have constructed a continuous linear operator from $W^{1, q}\left(\Omega^{0}\right)$ to $W^{1-\frac{1}{q}, q}\left(\Gamma^{\infty}\right)$, which extends the trace operator defined on $\mathcal{C}^{\infty}\left(\overline{\Omega^{0}}\right)$, and is unique by density. It is surjective because the trace operator from $W^{1, q}\left(\widehat{\Omega}^{0}\right)$ to $W^{1-\frac{1}{q}, q}\left(\Gamma^{\infty}\right)$ is surjective.

Remark 5. Of course, for two real numbers $1<q<q^{\prime}<2$, the restriction to $W^{1, q^{\prime}}\left(\Omega^{0}\right)$ of the trace operator defined above on $W^{1, q}\left(\Omega^{0}\right)$, coincides with the trace operator defined above on $W^{1, q^{\prime}}\left(\Omega^{0}\right)$, from the density of $\mathcal{C}^{\infty}\left(\overline{\Omega^{0}}\right)$ in both Sobolev spaces.

For any $q, 1<q<2$ and $u \in W^{1, q}\left(\Omega^{0}\right)$, we shall denote with $\left.u\right|_{\Gamma^{\infty}}$ the trace of $u$ on $\Gamma^{\infty}$ defined in Theorem 5 .

Proposition 2. Consider $p, q$ in $(1,2)$ such that $4 / 3<p, q$ which ensures that $W^{1-\frac{1}{p}, p}\left(\Gamma^{\infty}\right) \subset L^{2}\left(\Gamma^{\infty}\right)$ and $W^{1-\frac{1}{q}, q}\left(\Gamma^{\infty}\right) \subset L^{2}\left(\Gamma^{\infty}\right)$. For all $u \in W^{1, p}\left(\Omega^{0}\right)$, for all $g \in W^{1, q}\left(\Omega^{0}\right)$,

$$
\left.\left.\lim _{n \rightarrow \infty} \int_{\Gamma^{n}} u\right|_{\Gamma^{n}} g\right|_{\Gamma^{n}}=\left.\left.\frac{1}{3} \int_{\Gamma^{\infty}} u\right|_{\Gamma^{\infty}} g\right|_{\Gamma^{\infty}}
$$

Proof. We skip the proof for brevity. The main ingredient is the density of $C^{\infty}\left(\overline{\Omega^{0}}\right)$ in both Sobolev spaces.

For what follows, we define a continuous trace operator from $H^{1}\left(\Omega^{0}\right)$ on $L^{2}\left(\Gamma^{\infty}\right)$ :

Definition 1. We fix $r, 4 / 3<r<2$, and we have that $W^{1-\frac{1}{r}, r}\left(\Gamma^{\infty}\right)$ is (compactly) imbedded in $L^{2}\left(\Gamma^{\infty}\right)$. We call $i_{r}$ the imbedding from $H^{1}\left(\Omega^{0}\right)$ to $W^{1, r}\left(\Omega^{0}\right)$ and, for $u \in H^{1}\left(\Omega^{0}\right)$, we define $\gamma(u)=\left.\left(i_{r}(u)\right)\right|_{\Gamma^{\infty}}$. It is clear that $\gamma$ is a continuous operator from $H^{1}\left(\Omega^{0}\right)$ to $L^{2}\left(\Gamma^{\infty}\right)$, which extends the trace operator defined on $\mathcal{C}^{\infty}\left(\overline{\Omega^{0}}\right)$.

\section{Remark 6.}

- From the fact that $H^{1}\left(\Omega^{0}\right) \subset W^{1, q}\left(\Omega^{0}\right)$, for all $1<q<2$, and from Remark 5 , the definition of $\gamma$ does not depend on $r$.

- The operator $\gamma$ may not be the only continuous operator from $H^{1}\left(\Omega^{0}\right)$ to $L^{2}\left(\Gamma^{\infty}\right)$, extending the trace operator on $\mathcal{C}^{\infty}\left(\overline{\Omega^{0}}\right)$, because $\mathcal{C}^{\infty}\left(\overline{\Omega^{0}}\right)$ is not dense in $H^{1}\left(\Omega^{0}\right)$.

- The operator $\gamma$ has the following property: for all $g \in W^{1, p}\left(\Omega^{0}\right)$ with $4 / 3<p<2$, and for all $u \in H^{1}\left(\Omega^{0}\right)$,

$$
\left.\left.\lim _{n \rightarrow \infty} \int_{\Gamma^{n}} u\right|_{\Gamma^{n}} g\right|_{\Gamma^{n}}=\left.\frac{1}{3} \int_{\Gamma^{\infty}} \gamma(u) g\right|_{\Gamma^{\infty}} .
$$

- In the same manner, for any $q \geq 1$, it is possible to define a continuous operator from $H^{1}\left(\Omega^{0}\right)$ to $L^{q}\left(\Gamma^{\infty}\right)$, which extends the trace operator defined on $\mathcal{C}^{\infty}\left(\overline{\Omega^{0}}\right)$. 


\section{Poisson problems}

\subsection{Definition, existence and uniqueness results}

The goal of this section is to study some Poisson problems in $\Omega^{0}$ (the partial differential equation is $-\Delta w=0$ ) with

- Dirichlet boundary condition on $\Gamma^{0}$;

- homogeneous Neumann boundary condition on $\Sigma^{0}$;

- Neumann boundary condition on $\Gamma^{\infty}$.

Since the boundary of $\Omega^{0}$ is very irregular, a good way to give sense to these problems (especially to the Neumann boundary condition on $\Gamma^{\infty}$ ) is to use variational or weak formulations.

More precisely, take $g \in L^{2}\left(\Gamma^{\infty}\right)$ and $u \in H^{\frac{1}{2}}\left(\Gamma^{0}\right)$. We are interested in the variational problem: find $w \in H^{1}\left(\Omega^{0}\right)$ such that

$$
\begin{aligned}
\left.w\right|_{\Gamma^{0}} & =u \\
\int_{\Omega^{0}} \nabla w \cdot \nabla v & =\frac{1}{3} \int_{\Gamma^{\infty}} g \gamma(v), \quad \forall v \in \mathcal{V}\left(\Omega^{0}\right) .
\end{aligned}
$$

This function is a weak solution to

$$
\begin{aligned}
-\Delta w & =0, & & \text { in } \Omega^{0}, \\
w & =u, & & \text { on } \Gamma^{0}, \\
\frac{\partial w}{\partial n} & =0, & & \text { on } \Sigma^{0},
\end{aligned}
$$

with an additional Neumann condition on $\Gamma^{\infty}$ which can only be written in a variational setting (to the best of our knowledge).

All what follows can be generalized to the equation $-\operatorname{div}(\chi \nabla w)=0$, where $\chi$ is a symmetric and positive definite constant tensor.

Proposition 3. For $g \in L^{2}\left(\Gamma^{\infty}\right)$ and $u \in H^{\frac{1}{2}}\left(\Gamma^{0}\right)$, problem (32) has a unique solution.

Proof. The linear form $v \mapsto \int_{\Gamma \infty} g \gamma(v)$ is continuous on $H^{1}\left(\Omega^{0}\right)$. Therefore, from Theorem 1, problem (32) has a unique solution.

Remark 7. In view of the fourth point of Remark 6, it is possible to consider in (32) a more general Neumann datum $g$ on $\Gamma^{\infty}$, i.e. $g \in L^{p}\left(\Gamma^{\infty}\right)$ with $p>1$ arbitrary.

The following result says that the solution to (32) can be approximated by solving boundary value problems in the polygonal domains $Y^{n}, n \rightarrow \infty$, with Neumann boundary condition on $\partial Y^{n} \backslash \Gamma^{0}$ :

Proposition 4. Assume that $g \in W^{1-\frac{1}{p}, p}\left(\Gamma^{\infty}\right)$, for some $p, 4 / 3<p<2$. Then $g \in L^{2}\left(\Gamma^{\infty}\right)$ and there exists $\tilde{g} \in W^{1, p}\left(\widehat{\Omega}^{0}\right)$ such that $g=\left.\tilde{g}\right|_{\Gamma^{\infty}}$. Take $u \in H^{\frac{1}{2}}\left(\Gamma^{0}\right)$. Let $w$ be the solution to (32) and $w_{n} \in H^{1}\left(Y^{n}\right)$ be the solution to:

we have

$$
\begin{aligned}
\left.w_{n}\right|_{\Gamma^{0}} & =u \\
\int_{Y^{n}} \nabla w_{n} \cdot \nabla v & =\left.\left.\int_{\Gamma^{n+1}} \tilde{g}\right|_{\Gamma^{n+1}} v\right|_{\Gamma^{n+1}}, \quad \forall v \in \mathcal{V}\left(Y^{n}\right)
\end{aligned}
$$

$$
\lim _{n \rightarrow \infty}\left\|\left.w\right|_{Y^{n}}-w_{n}\right\|_{H^{1}\left(Y^{n}\right)}=0 .
$$

Proof. Calling $e_{n}$ the error $e_{n}=\left.w\right|_{Y^{n}}-w_{n} \in \mathcal{V}\left(Y^{n}\right)$, we see that $\forall v \in \mathcal{V}\left(\Omega^{0}\right)$,

$$
\int_{Y^{n}} \nabla e_{n} \cdot \nabla v=\left(\frac{1}{3} \int_{\Gamma^{\infty}} g \gamma(v)-\left.\left.\int_{\Gamma^{n+1}} \tilde{g}\right|_{\Gamma^{n+1}} v\right|_{\Gamma^{n+1}}\right)-\int_{\Omega^{n+1}} \nabla w \cdot \nabla v
$$

From Remark $6, \frac{1}{3} \int_{\Gamma^{\infty}} g \gamma(v)=\left.\left.\lim _{n \rightarrow \infty} \int_{\Gamma^{n+1}} \tilde{g}\right|_{\Gamma^{n+1}} v\right|_{\Gamma^{n+1}}$, so the first term in the right hand side tends to zero. Moreover, Proposition 1 shows that for all $q, 1<q<p$, the function $\tilde{g} v$ belongs to $W^{1, q}\left(\Omega^{0}\right)$, and it is 
easy to check by a scaling argument that, for all $m<m^{\prime}$,

$$
\left.\left|\int_{\Gamma^{m+1}} \tilde{g}\right|_{\Gamma^{m+1}} v\right|_{\Gamma^{m+1}}-\left.\left.\int_{\Gamma^{m^{\prime}+1}} \tilde{g}\right|_{\Gamma^{m^{\prime}+1}} v\right|_{\Gamma^{m^{\prime}+1}} \mid \lesssim 2^{m \frac{1-q}{q}}\|\nabla(\tilde{g} v)\|_{L^{q}\left(Y^{m^{\prime}} \backslash Y^{m}\right)} .
$$

Since $\tilde{g}$ is fixed, this implies that

$$
\left.\left|\int_{\Gamma^{m+1}} \tilde{g}\right|_{\Gamma^{m+1}} v\right|_{\Gamma^{m+1}}-\left.\left.\int_{\Gamma^{m^{\prime}+1}} \tilde{g}\right|_{\Gamma^{m^{\prime}+1}} v\right|_{\Gamma^{m^{\prime}+1}} \mid \lesssim 2^{m \frac{1-q}{q}}\|v\|_{H^{1}\left(\Omega^{0}\right)}
$$

Thus, the sequence of continuous linear forms on $\mathcal{V}\left(\Omega^{0}\right):\left.\left.v \mapsto \int_{\Gamma^{n+1}} \tilde{g}\right|_{\Gamma^{n+1}} v\right|_{\Gamma^{n+1}}$ is a Cauchy sequence in the dual of $\mathcal{V}\left(\Omega^{0}\right)$, therefore,

$$
\lim _{n \rightarrow \infty} \sup _{v \in \mathcal{V}\left(\Omega^{0}\right), v \neq 0} \frac{\left.\left|\frac{1}{3} \int_{\Gamma^{\infty}} g \gamma(v)-\int_{\Gamma^{n+1}} \tilde{g}\right|_{\Gamma^{n+1}} v\right|_{\Gamma^{n+1}} \mid}{\|v\|_{H^{1}\left(\Omega^{0}\right)}}=0 .
$$

We also have that

$$
\lim _{n \rightarrow \infty} \sup _{v \in \mathcal{V}\left(\Omega^{0}\right), v \neq 0} \frac{\int_{\Omega^{n+1}} \nabla w \cdot \nabla v}{\|v\|_{H^{1}\left(\Omega^{0}\right)}}=0 .
$$

From (34), (35) and (36), we deduce the desired result.

Remark 8. Questioning the intrinsic character of (32) is natural since there may be other continuous trace operators $\gamma$ from $H^{1}\left(\Omega^{0}\right)$ to $L^{2}\left(\Gamma^{\infty}\right)$. Proposition 4 shows that if $g=\left.\tilde{g}\right|_{\Gamma_{\infty}}$ with $\tilde{g} \in W^{1, p}\left(\widehat{\Omega}^{0}\right)$, for some $p$, $4 / 3<p<2$, then the solution to (32) can be approximated by solving the boundary value problems in $Y^{n}$ :

$$
\begin{array}{rlrl}
-\Delta w_{n} & =0, & & \text { in } Y^{n}, \\
w_{n} & =u, & & \text { on } \Gamma^{0}, \\
\frac{\partial w_{n}}{\partial n}=\tilde{g}, & & \text { on } \Gamma^{n+1}, \\
\frac{\partial w_{n}}{\partial n}=0, & & \text { on } \partial Y^{n} \backslash\left(\Gamma^{0} \cup \Gamma^{n+1}\right) .
\end{array}
$$

This gives an intrinsic meaning to problem (32), under the last assumption on $g$.

\section{TRANSPARENT BOUNDARY CONDITIONS FOR PROBLEM (32)}

\subsection{General orientation}

The following is part of a program consisting of approximating the restriction to the truncated domain $Y^{n}$ of $w$, the solution to (32), to the best possible accuracy. This is important, because, in numerical simulations, it is not possible to completely represent the domain $\Omega^{0}$, for this would imply infinite memory and computing time. For example, one can only look for the restriction of $w$ to $Y^{0}$, (i.e. $\left.n=0\right)$.

In the following, we will restrict ourselves to the simpler case when $g=0$ (homogeneous Neumann boundary conditions on $\left.\Gamma^{\infty}\right)$. In this case, it is possible to find the restriction of $w$ to $Y^{n}$, but for that, we need to use nonlocal operators on $\Gamma^{\sigma}, \sigma \in \mathcal{A}_{n+1}$. We will see that these operators can be called Dirichlet to Neumann operators. They will be computed, or more precisely approximated up to an arbitrary accuracy, by taking advantage of the self-similarity in the geometry. The new boundary conditions on $\Gamma^{n+1}$ can be called transparent boundary conditions.

Moreover, as we shall see in the beginning of Section 5.2.3, solving the new boundary value problem in $Y^{n}$ is equivalent to successively solving $1+2+\cdots+2^{n}$ boundary value problems in $Y^{0}$, (this is very useful for numerical simulations). In this paper, we stay at the continuous level: numerical methods and results are presented in $[2]$. 
The general case $g \neq 0$ can be handled too: the method uses the fact that if $g$ is a Haar wavelet on $\Gamma^{\infty}$ (working with Haar wavelets is very natural with the chosen geometry), then the restriction of $w$ to $Y^{n}$ can be computed with an arbitrary accuracy by using a transparent boundary condition, obtained by taking advantage of the self-similarity. Then, for a general $g$, we expand $g$ on the basis of Haar wavelets, and we use the linearity to compute $w$. This program, in the case $g \neq 0$, is discussed in [1], since its complete description is too long for the present paper. In [1], numerical results are presented too.

Here, we focus on the case when $g=0$.

\subsection{The case when the Neumann data on $\Gamma^{\infty}$ is zero}

\subsubsection{Harmonic lifting of functions defined on $\Gamma^{0}$}

For a function $u \in H^{\frac{1}{2}}\left(\Gamma^{0}\right)$, we define the harmonic lifting $\mathcal{H}^{0}(u) \in H^{1}\left(\Omega^{0}\right)$ of $u$ by: the trace of $\mathcal{H}^{0}(u)$ on $\Gamma^{0}$ is $u$, and for all $v \in \mathcal{V}\left(\Omega^{0}\right)$,

$$
\int_{\Omega^{0}} \nabla \mathcal{H}^{0}(u) \cdot \nabla v=0 .
$$

It is clear that $\mathcal{H}^{0}$ is a bounded operator from $H^{\frac{1}{2}}\left(\Gamma^{0}\right)$ to $H^{1}\left(\Omega^{0}\right)$.

Similarly, for an integer $n>0$ and for $\sigma \in \mathcal{A}_{n}$, one can define the lifting operator $\mathcal{H}^{\sigma}$ from $H^{\frac{1}{2}}\left(\Gamma^{\sigma}\right)$ to $H^{1}\left(\Omega^{\sigma}\right)$ : for all $u \in H^{\frac{1}{2}}\left(\Gamma^{\sigma}\right)$, the trace of $\mathcal{H}^{\sigma}(u)$ on $\Gamma^{\sigma}$ is $u$ and for all $v \in \mathcal{V}\left(\Omega^{\sigma}\right), \int_{\Omega^{\sigma}} \nabla \mathcal{H}^{\sigma}(u) \cdot \nabla v=0$. It is easy to verify that, for all $u \in H^{\frac{1}{2}}\left(\Gamma^{\sigma}\right)$,

$$
\mathcal{H}^{\sigma}(u) \circ \mathcal{M}_{\sigma}\left(F_{1}, F_{2}\right)=\mathcal{H}^{0}\left(u \circ \mathcal{M}_{\sigma}\left(F_{1}, F_{2}\right)\right)
$$

The following result will be useful to study the asymptotic behavior of $\left\|\nabla \mathcal{H}^{0}(u)\right\|_{L^{2}\left(\Omega^{n}\right)}$ as $n$ tends to $\infty$.

Lemma 3. There exists a positive constant $\mathrm{C}$ such that, for all $u \in H^{\frac{1}{2}}\left(\Gamma^{0}\right)$,

$$
\left\|\nabla \mathcal{H}^{0}(u)\right\|_{L^{2}\left(Y^{0}\right)} \geq \mathrm{C}\left\|\nabla \mathcal{H}^{0}(u)\right\|_{L^{2}\left(\Omega^{0}\right)}
$$

Proof. It is enough to prove $(39)$ for all $u \in H^{\frac{1}{2}}\left(\Gamma^{0}\right)$ such that $\int_{\Gamma^{0}} u=0$, because $\mathcal{H}^{0}\left(1_{\Gamma^{0}}\right)=1_{\Omega^{0}}$.

From the classical Poincaré-Wiertinger inequality in $Y^{0}$ :

$$
\|v\|_{L^{2}\left(Y^{0}\right)} \lesssim\|\nabla v\|_{L^{2}\left(Y^{0}\right)}, \quad \forall v \in H^{1}\left(Y^{0}\right) \text { s.t. }\left.\int_{\Gamma^{0}} v\right|_{\Gamma^{0}}=0
$$

we deduce that $\|u\|_{H^{\frac{1}{2}}\left(\Gamma^{0}\right)} \lesssim\left\|\nabla \mathcal{H}^{0}(u)\right\|_{L^{2}\left(Y^{0}\right)}$. On the other hand, from the continuity of $\mathcal{H}^{0}$, we have that $\left\|\nabla \mathcal{H}^{0}(u)\right\|_{L^{2}\left(\Omega^{0}\right)} \lesssim\|u\|_{H^{\frac{1}{2}}\left(\Gamma^{0}\right)}$. The desired result follows from the last two estimates.

Lemma 4. There exists a constant $\rho<1$ such that for all $u \in H^{\frac{1}{2}}\left(\Gamma^{0}\right)$,

$$
\int_{\Omega^{1}}\left|\nabla \mathcal{H}^{0}(u)\right|^{2} \leq \rho \int_{\Omega^{0}}\left|\nabla \mathcal{H}^{0}(u)\right|^{2} .
$$

Proof. The result is a direct consequence of Lemma 3.

Theorem 6. For all $u \in H^{\frac{1}{2}}\left(\Gamma^{0}\right)$,

$$
\int_{\Omega^{N}}\left|\nabla \mathcal{H}^{0}(u)\right|^{2} \leq \rho^{N} \int_{\Omega^{0}}\left|\nabla \mathcal{H}^{0}(u)\right|^{2},
$$

where the constant $\rho<1$ has been introduced in Lemma 4 . 
Proof. The desired result will be proved once we have established that

$$
\int_{\Omega^{n+1}}\left|\nabla \mathcal{H}^{0}(u)\right|^{2} \leq \rho \int_{\Omega^{n}}\left|\nabla \mathcal{H}^{0}(u)\right|^{2} .
$$

For that, we realize that for all $\sigma \in \mathcal{A}_{n}$, the function $\left.\mathcal{H}^{0}(u)\right|_{\Omega^{\sigma}} \circ \mathcal{M}_{\sigma}\left(F_{1}, F_{2}\right)$ defined on $\Omega^{0}$ is the harmonic lifting of a function in $H^{\frac{1}{2}}\left(\Gamma^{0}\right)$, namely

$$
\left.\mathcal{H}^{0}(u)\right|_{\Omega^{\sigma}} \circ \mathcal{M}_{\sigma}\left(F_{1}, F_{2}\right)=\mathcal{H}^{0}\left(\left.\mathcal{H}^{0}(u)\right|_{\Gamma^{\sigma}} \circ \mathcal{M}_{\sigma}\left(F_{1}, F_{2}\right)\right) .
$$

From this, we can use (40), and see that

$$
\int_{\Omega^{1}}\left|\nabla\left(\left.\mathcal{H}^{0}(u)\right|_{\Omega^{\sigma}} \circ \mathcal{M}_{\sigma}\left(F_{1}, F_{2}\right)\right)\right|^{2} \leq \rho \int_{\Omega^{0}}\left|\nabla\left(\left.\mathcal{H}^{0}(u)\right|_{\Omega^{\sigma}} \circ \mathcal{M}_{\sigma}\left(F_{1}, F_{2}\right)\right)\right|^{2},
$$

and performing the change of variables $y=\mathcal{M}_{\sigma}\left(F_{1}, F_{2}\right)(x)$, we obtain

$$
\int_{\mathcal{M}_{\sigma}\left(F_{1}, F_{2}\right)\left(\Omega^{1}\right)}\left|\nabla \mathcal{H}^{0}(u)\right|^{2} \leq \rho \int_{\Omega^{\sigma}}\left|\nabla \mathcal{H}^{0}(u)\right|^{2} .
$$

Summing over $\sigma \in \mathcal{A}_{n}$, we get (42). From (42), the desired result is proved by induction.

Remark 9. Theorem 6 can be explained intuitively by the fact that when we go toward the small structures of the ramified domain, diffusion becomes more and more important, and the solution to the Poisson problem gets exponentially dampened. As we shall see later, the same phenomenon occurs with the Helmholtz equation.

From the general theory of boundary value problems, see [7] for example, we have the following regularity:

Lemma 5 (local regularity). For all $u \in H^{\frac{1}{2}}\left(\Gamma^{0}\right)$, for all open bounded domain $\mathcal{O}$ strongly contained in $\mathbb{R} \times(0,6)$, and for all $\epsilon, 0<\epsilon<\frac{5}{3}$, the restriction of $\mathcal{H}^{0}(u)$ to $\Omega^{0} \cap \mathcal{O}$ belongs to $H^{\frac{5}{3}-\epsilon}\left(\Omega^{0} \cap \mathcal{O}\right)$.

\subsubsection{The Dirichlet-Neumann operator and transparent boundary conditions}

Call $\left(H^{\frac{1}{2}}\left(\Gamma^{0}\right)\right)^{\prime}$ the topological dual space of $H^{\frac{1}{2}}\left(\Gamma^{0}\right)$ and consider the Dirichlet-Neumann operator $T^{0}$ : $H^{\frac{1}{2}}\left(\Gamma^{0}\right) \mapsto\left(H^{\frac{1}{2}}\left(\Gamma^{0}\right)\right)^{\prime}$

We remark that

$$
\left\langle T^{0} u, v\right\rangle=\int_{\Omega^{0}} \nabla \mathcal{H}^{0}(u) \cdot \nabla \mathcal{H}^{0}(v)
$$

$$
\left\langle T^{0} u, v\right\rangle=\int_{\Omega^{0}} \nabla \mathcal{H}^{0}(u) \cdot \nabla \tilde{v}
$$

for any function $\tilde{v} \in H^{1}\left(\Omega^{0}\right)$ such that $\left.\tilde{v}\right|_{\Gamma^{0}}=v$.

In fact, $T^{0} u$ is the normal derivative of $\mathcal{H}^{0}(u)$ on $\Gamma^{0}$. This is why $T^{0}$ is called a Dirichlet-Neumann operator.

The operator $T^{0}$ is bounded, self-adjoint and positive semi-definite. It is clear that $T^{0} 1=0$. Call $V$ the closed subspace of $H^{\frac{1}{2}}\left(\Gamma^{0}\right)$ :

$$
V=\left\{v \in H^{\frac{1}{2}}\left(\Gamma^{0}\right), \int_{\Gamma^{0}} v=0\right\} .
$$

From (14) and from (17), we see that $T^{0}$ is coercive on $V$, i.e. there exists a positive constant $\alpha$ such that

$$
\forall v \in V, \quad\left\langle T^{0} v, v\right\rangle \geq \alpha\|v\|_{H^{\frac{1}{2}}\left(\Gamma^{0}\right)}^{2} .
$$


Similarly, for $\sigma \in \mathcal{A}_{n}$, one can define the operators $T^{\sigma}$, from $H^{\frac{1}{2}}\left(\Gamma^{\sigma}\right)$ (see (8) and (10)) to their respective duals by $\left\langle T^{\sigma} u, v\right\rangle=\int_{\Omega^{\sigma}} \nabla \mathcal{H}^{\sigma}(u) \cdot \nabla \mathcal{H}^{\sigma}(v)=\int_{\Omega^{\sigma}} \nabla \mathcal{H}^{\sigma}(u) \cdot \nabla \tilde{v}$, for any function $\tilde{v} \in H^{1}\left(\Omega^{\sigma}\right)$ such that $\left.\tilde{v}\right|_{\Gamma^{\sigma}}=v$. From the self-similarity of $\Omega^{0}$ and (38), we have that

$$
\forall u, v \in H^{\frac{1}{2}}\left(\Gamma^{\sigma}\right), \quad\left\langle T^{\sigma} u, v\right\rangle=\left\langle T^{0}\left(u \circ \mathcal{M}_{\sigma}\left(F_{1}, F_{2}\right)\right),\left(v \circ \mathcal{M}_{\sigma}\left(F_{1}, F_{2}\right)\right)\right\rangle,
$$

where the duality pairing on the left (resp. right) hand side of $(47)$ is the duality $\left(H^{\frac{1}{2}}\left(\Gamma^{\sigma}\right)\right)^{\prime}-H^{\frac{1}{2}}\left(\Gamma^{\sigma}\right)($ resp. $\left.\left(H^{\frac{1}{2}}\left(\Gamma^{0}\right)\right)^{\prime}-H^{\frac{1}{2}}\left(\Gamma^{0}\right)\right)$.

Lemma 6. For all $u \in H^{\frac{1}{2}}\left(\Gamma^{0}\right)$, for $n \geq 1$, the restriction of $\mathcal{H}^{0}(u)$ to $Y^{n-1}$ is the solution to the following boundary value problem: find $\hat{u} \in H^{1}\left(Y^{n-1}\right)$ such that $\left.\hat{u}\right|_{\Gamma^{0}}=u$ and $\forall v \in \mathcal{V}\left(Y^{n-1}\right)$,

$$
\int_{Y^{n-1}} \nabla \hat{u} \cdot \nabla v+\sum_{\sigma \in \mathcal{A}_{n}}\left\langle T^{0}\left(\left.\hat{u}\right|_{\Gamma^{\sigma}} \circ \mathcal{M}_{\sigma}\left(F_{1}, F_{2}\right)\right),\left.v\right|_{\Gamma^{\sigma}} \circ \mathcal{M}_{\sigma}\left(F_{1}, F_{2}\right)\right\rangle=0 .
$$

Furthermore, $\forall v \in H^{1}\left(Y^{n-1}\right)$,

$$
\begin{aligned}
\left\langle T^{0} u,\left.v\right|_{\Gamma^{0}}\right\rangle & =\int_{Y^{n-1}} \nabla \hat{u} \cdot \nabla v+\sum_{\sigma \in \mathcal{A}_{n}}\left\langle\left. T^{\sigma} \hat{u}\right|_{\Gamma^{\sigma}},\left.v\right|_{\Gamma^{\sigma}}\right\rangle \\
& =\int_{Y^{n-1}} \nabla \hat{u} \cdot \nabla v+\sum_{\sigma \in \mathcal{A}_{n}}\left\langle T^{0}\left(\left.\hat{u}\right|_{\Gamma^{\sigma}} \circ \mathcal{M}_{\sigma}\left(F_{1}, F_{2}\right)\right),\left.v\right|_{\Gamma^{\sigma}} \circ \mathcal{M}_{\sigma}\left(F_{1}, F_{2}\right)\right\rangle .
\end{aligned}
$$

Proof. Follows from (38) and (47).

Remark 10. Note that the boundary value problem (48) is well posed because the bilinear form on the left hand side is continuous, symmetric and coercive on $\mathcal{V}\left(Y^{n-1}\right)$.

We see that, for a fixed arbitrary integer $n>0$ (for example $n=1$ ), once the nonlocal operator $T^{0}$ is known, the restriction of $\mathcal{H}^{0}(u)$ to the truncated domain $Y^{n-1}$ can be found by solving the boundary value problem (48) in $Y^{n-1}$ with a boundary condition involving $T^{0}$. It is important to understand that (48) is a Poisson problem in $Y^{n-1}$, with a Dirichlet boundary condition on $\Gamma^{0}$, a homogeneous Neumann condition on $\partial Y^{n-1} \backslash\left(\Gamma^{0} \cup \Gamma^{n}\right)$, and for each $\sigma \in \mathcal{A}_{n}$,

$$
\frac{\partial \hat{u}}{\partial n}+\left.T^{\sigma} \hat{u}\right|_{\Gamma^{\sigma}}=0, \quad \text { on } \Gamma^{\sigma},
$$

and all the operators $T^{\sigma}, \sigma \in \mathcal{A}_{n}$ are obtained readily from $T^{0}$ by (47). Equation (50) is a nonlocal boundary condition, called a transparent boundary condition. Furthermore, solving (48) is equivalent to successively solving $1+2+\cdots+2^{n-1}$ boundary value problems in $Y^{0}$ : indeed, an algorithm for solving (48) is as follows:

\section{Algorithm 1.}

- Loop: for $p=0$ to $n-1$,

- Loop : for $\sigma \in \mathcal{A}_{p}$, (at this point, $\left.\hat{u}\right|_{\Gamma^{\sigma}}$ is known)

* Solve the boundary value problem in $Y^{0}$ : find $w \in H^{1}\left(Y^{0}\right)$ such that $\left.w\right|_{\Gamma^{0}}=\left.\hat{u}\right|_{\Gamma^{\sigma}} \circ$ $\mathcal{M}_{\sigma}\left(F_{1}, F_{2}\right)$ and

$$
\int_{Y^{0}} \nabla w \cdot \nabla v+\sum_{i=1}^{2}\left\langle T^{0}\left(\left.w\right|_{F_{i}\left(\Gamma^{0}\right)} \circ F_{i}\right),\left.v\right|_{F_{i}\left(\Gamma^{0}\right)} \circ F_{i}\right\rangle=0, \quad \forall v \in \mathcal{V}\left(Y^{0}\right) .
$$

* Set $\left.\hat{u}\right|_{\mathcal{M}_{\sigma}\left(F_{1}, F_{2}\right)\left(Y^{0}\right)}=w \circ\left(\mathcal{M}_{\sigma}\left(F_{1}, F_{2}\right)\right)^{-1}$. 
Let us stress the fact that, in the numerical simulations of $\left.\mathcal{H}^{0}(u)\right|_{Y^{n-1}}$, Algorithm 1 saves solving discrete boundary value problems in the domain $Y^{n-1}$, which is complicated when $n$ is large. This is why the transparent boundary condition is well suited for numerical simulations, as soon as $T^{0}$ or a good approximation to $T^{0}$ is known. This program has been carried out successfully with finite elements and the numerical method and its results are presented in [2], for both the Laplace and Helmholtz equations.

\subsubsection{Approximation to the Dirichlet-Neumann operator}

Orientation. We have seen above that if $T^{0}$ or a good approximation of $T^{0}$ is available, then the restriction of $\mathcal{H}^{0}(u)$ to $Y^{n-1}$ can be approximated by a standard discrete method for (48), which consists of successively solving $1+2+\cdots+2^{n-1}$ discrete boundary value problems in $Y^{0}$ with a transparent boundary condition on $\Gamma^{1}$.

There remains the computation of $T^{0}$, or at least its accurate approximation: for that, we will make use of (49), in the case $n=1$.

The operator $T^{0}$ can be constructed as the limit of an inductive sequence. Lemma 6 , in the case $n=1$, leads us to introduce the cone $\mathbb{O}$ of self adjoint, positive semi-definite, bounded linear operators from $H^{\frac{1}{2}}\left(\Gamma^{0}\right)$ to its dual, vanishing on the constants, and the mapping $\mathbb{M}: \mathbb{O} \mapsto \mathbb{O}$ defined as follows: for $Z \in \mathbb{O}$, define $\mathbb{M}(Z)$ by

$$
\forall u \in H^{\frac{1}{2}}\left(\Gamma^{0}\right), \forall v \in H^{1}\left(Y^{0}\right), \quad\left\langle\mathbb{M}(Z) u,\left.v\right|_{\Gamma^{0}}\right\rangle=\int_{Y^{0}} \nabla \hat{u} \cdot \nabla v+\sum_{i=1}^{2}\left\langle Z\left(\left.\hat{u}\right|_{F_{i}\left(\Gamma^{0}\right)} \circ F_{i}\right),\left.v\right|_{F_{i}\left(\Gamma^{0}\right)} \circ F_{i}\right\rangle
$$

where $\hat{u} \in H^{1}\left(Y^{0}\right)$ is such that $\left.\hat{u}\right|_{\Gamma^{0}}=u$ and

$$
\forall v \in \mathcal{V}\left(Y^{0}\right), \quad \int_{Y^{0}} \nabla \hat{u} \cdot \nabla v+\sum_{i=1}^{2}\left\langle Z\left(\left.\hat{u}\right|_{F_{i}\left(\Gamma^{0}\right)} \circ F_{i}\right),\left.v\right|_{F_{i}\left(\Gamma^{0}\right)} \circ F_{i}\right\rangle=0 .
$$

Remark 11. From the definition of $\mathbb{M}$, it can be seen, (see (54) and (55) below), that for all $p \geq 1$, if $w$ satisfies the Poisson problem $\Delta w=0$ in $Y^{p-1}$, with $\frac{\partial w}{\partial n}=0$ on $\partial Y^{p-1} \backslash\left(\Gamma^{0} \cup \Gamma^{p}\right)$, and with $\left.\frac{\partial w}{\partial n}\right|_{\Gamma^{\sigma}} \circ \mathcal{M}_{\sigma}\left(F_{1}, F_{2}\right)=$ $-2^{p} Z\left(\left.w\right|_{\Gamma^{\sigma}} \circ \mathcal{M}_{\sigma}\left(F_{1}, F_{2}\right)\right), \sigma \in \mathcal{A}_{p}$, then $\left.\frac{\partial w}{\partial n}\right|_{\Gamma^{0}}=\mathbb{M}^{p}(Z)\left(\left.w\right|_{\Gamma^{0}}\right)$.

Lemma 6 shows that $T^{0}$ is a fixed point of $\mathbb{M}$. In fact, we have the

Theorem 7. The operator $T^{0}$ is the unique fixed point of $\mathbb{M}$. Moreover, for all $Z \in \mathbb{O}$, there exists a positive constant $\mathrm{C}$ such that, for all $p \geq 0$,

$$
\left\|\mathbb{M}^{p}(Z)-T^{0}\right\| \leq \mathrm{C} \rho^{\frac{p}{4}}
$$

where $\rho, 0<\rho<1$ is the constant appearing in Lemma 4 .

Proof. It is easy to check by induction that

$$
\begin{aligned}
& \forall u \in H^{\frac{1}{2}}\left(\Gamma^{0}\right), \forall v \in H^{1}\left(Y^{p-1}\right), \\
& \left\langle\mathbb{M}^{p}(Z) u,\left.v\right|_{\Gamma^{0}}\right\rangle=\int_{Y^{p-1}} \nabla \hat{u} \cdot \nabla v+\sum_{\sigma \in \mathcal{A}_{p}}\left\langle Z\left(\left.\hat{u}\right|_{\Gamma^{\sigma}} \circ \mathcal{M}_{\sigma}\left(F_{1}, F_{2}\right)\right),\left(\left.v\right|_{\Gamma^{\sigma}} \circ \mathcal{M}_{\sigma}\left(F_{1}, F_{2}\right)\right)\right\rangle,
\end{aligned}
$$

where $\hat{u} \in H^{1}\left(Y^{p-1}\right)$ is such that $\left.\hat{u}\right|_{\Gamma^{0}}=u$ and

$$
\forall v \in \mathcal{V}\left(Y^{p-1}\right), \quad \int_{Y^{p-1}} \nabla \hat{u} \cdot \nabla v+\sum_{\sigma \in \mathcal{A}_{p}}\left\langle Z\left(\left.\hat{u}\right|_{\Gamma^{\sigma}} \circ \mathcal{M}_{\sigma}\left(F_{1}, F_{2}\right)\right),\left(\left.v\right|_{\Gamma^{\sigma}} \circ \mathcal{M}_{\sigma}\left(F_{1}, F_{2}\right)\right)\right\rangle=0 .
$$


Therefore, for $\phi \in H^{\frac{1}{2}}\left(\Gamma^{0}\right)$,

$$
\begin{aligned}
\left\langle\left(\mathbb{M}^{p}(Z)-T^{0}\right) u, \phi\right\rangle & =I+I I+I I I, \\
I & =\int_{Y^{p-1}} \nabla\left(\hat{u}-\mathcal{H}^{0}(u)\right) \cdot \nabla \mathcal{H}^{0}(\phi), \\
I I & =\sum_{\sigma \in \mathcal{A}_{p}}\left\langle Z\left(\left.\left(\hat{u}-\mathcal{H}^{0}(u)\right)\right|_{\Gamma^{\sigma}} \circ \mathcal{M}_{\sigma}\left(F_{1}, F_{2}\right)\right),\left.\mathcal{H}^{0}(\phi)\right|_{\Gamma^{\sigma}} \circ \mathcal{M}_{\sigma}\left(F_{1}, F_{2}\right)\right\rangle, \\
I I I & \left.=\sum_{\sigma \in \mathcal{A}_{p}}\left\langle\left.\left(Z-T^{0}\right)\left(\mathcal{H}^{0}(u)\right)\right|_{\Gamma^{\sigma}} \circ \mathcal{M}_{\sigma}\left(F_{1}, F_{2}\right)\right),\left.\mathcal{H}^{0}(\phi)\right|_{\Gamma^{\sigma}} \circ \mathcal{M}_{\sigma}\left(F_{1}, F_{2}\right)\right\rangle .
\end{aligned}
$$

In order to estimate $I, I I$ and $I I I$, we introduce $\mathcal{H}^{p}(\hat{u}) \in H^{1}\left(\Omega^{0}\right)$ defined by

$$
\mathcal{H}^{p}(\hat{u})=\left\{\begin{aligned}
\hat{u} & \text { in } Y^{p-1}, \\
\text { the harmonic lifting of }\left.\hat{u}\right|_{\Gamma^{p}} & \text { in } \Omega^{p}
\end{aligned}\right.
$$

It can be proved that for a constant $\mathrm{C}$ independent of $p,\left\|\mathcal{H}^{p}(\hat{u})\right\|_{H^{1}\left(\Omega^{0}\right)} \leq \mathrm{C}\|\hat{u}\|_{H^{1}\left(Y^{p-1}\right)}$. Then

$$
\begin{aligned}
& \int_{Y^{p-1}}\left|\nabla\left(\hat{u}-\mathcal{H}^{0}(u)\right)\right|^{2}+\sum_{\sigma \in \mathcal{A}_{p}}\left\langle Z\left(\left.\left(\hat{u}-\mathcal{H}^{0}(u)\right)\right|_{\Gamma^{\sigma}} \circ \mathcal{M}_{\sigma}\left(F_{1}, F_{2}\right)\right),\left.\left(\hat{u}-\mathcal{H}^{0}(u)\right)\right|_{\Gamma^{\sigma}} \circ \mathcal{M}_{\sigma}\left(F_{1}, F_{2}\right)\right\rangle \\
& =-\int_{Y^{p-1}} \nabla\left(\mathcal{H}^{p}(\hat{u})-\mathcal{H}^{0}(u)\right) \cdot \nabla \mathcal{H}^{0}(u)-\sum_{\sigma \in \mathcal{A}_{p}}\left\langle Z\left(\left.\left(\mathcal{H}^{0}(u)\right)\right|_{\Gamma^{\sigma}} \circ \mathcal{M}_{\sigma}\left(F_{1}, F_{2}\right)\right),\left.\left(\hat{u}-\mathcal{H}^{0}(u)\right)\right|_{\Gamma^{\sigma}} \circ \mathcal{M}_{\sigma}\left(F_{1}, F_{2}\right)\right\rangle \\
& =\int_{\Omega^{p}} \nabla\left(\mathcal{H}^{p}(\hat{u})-\mathcal{H}^{0}(u)\right) \cdot \nabla \mathcal{H}^{0}(u)-\sum_{\sigma \in \mathcal{A}_{p}}\left\langle Z\left(\left.\left(\mathcal{H}^{0}(u)\right)\right|_{\Gamma^{\sigma}} \circ \mathcal{M}_{\sigma}\left(F_{1}, F_{2}\right)\right),\left.\left(\hat{u}-\mathcal{H}^{0}(u)\right)\right|_{\Gamma^{\sigma}} \circ \mathcal{M}_{\sigma}\left(F_{1}, F_{2}\right)\right\rangle \\
& \lesssim\left(\int_{\Omega^{p}}\left|\nabla \mathcal{H}^{0}(u)\right|^{2}\right)^{\frac{1}{2}}\left(\int_{\Omega^{p}}\left|\nabla \mathcal{H}^{p}(\hat{u})-\nabla \mathcal{H}^{0}(u)\right|^{2}\right)^{\frac{1}{2}} \\
& \lesssim \rho^{\frac{p}{2}}\left\|\nabla \mathcal{H}^{0}(u)\right\|_{L^{2}\left(\Omega^{0}\right)}\left(\left\|\nabla\left(\mathcal{H}^{0}(u)\right)\right\|_{L^{2}\left(\Omega^{p}\right)}+\left\|\nabla\left(\mathcal{H}^{p}(\hat{u})\right)\right\|_{L^{2}\left(\Omega^{p}\right)}\right) \\
& \lesssim \rho^{\frac{p}{2}}\left\|\nabla \mathcal{H}^{0}(u)\right\|_{L^{2}\left(\Omega^{0}\right)}\left(\left\|\mathcal{H}^{0}(u)\right\|_{H^{1}\left(\Omega^{0}\right)}+\|\hat{u}\|_{H^{1}\left(Y^{p-1}\right)}\right) \\
& \lesssim \rho^{\frac{p}{2}}\|u\|_{H^{\frac{1}{2}}\left(\Gamma^{0}\right)}^{2},
\end{aligned}
$$

where the constant hidden in the sign $\lesssim$ depends on $Z$.

Because of the estimate written above, $|I+I I| \lesssim \rho^{\frac{p}{4}}\|u\|_{H^{\frac{1}{2}}\left(\Gamma^{0}\right)}\|\phi\|_{H^{\frac{1}{2}}\left(\Gamma^{0}\right)}$. Estimate (41) implies that $|I I I| \lesssim \rho^{p}\|u\|_{H^{\frac{1}{2}\left(\Gamma^{0}\right)}}\|\phi\|_{H^{\frac{1}{2}\left(\Gamma^{0}\right)}}$. Estimate (53) is proved. As a consequence, $T^{0}$ is the unique fixed point of $\mathbb{M}$.

As a consequence of Theorem 7, it is simple to prove that for fixed $n$, if in Algorithm $1, T^{0}$ is replaced with $\mathbb{M}^{p}(Z)$, then the error on $\left.\mathcal{H}^{0}(u)\right|_{Y^{n-1}}$ decays at least like $\rho^{\frac{p}{4}}$ as $p$ tends to infinity. This behavior is observed in the numerical tests presented in [2]. 


\section{Propagation problems and transparent Boundary CONDitions}

The goal of this section is to study the weak solutions of the Helmholtz equation that is satisfied by a class of time-harmonic waves in the domain $\Omega^{0}$. The analysis of the problem uses the compact imbedding of $H^{1}\left(\Omega^{0}\right)$ in $L^{2}\left(\Omega^{0}\right)$, see Theorem 2, and Fredholm's alternative. Next, as in Section 5, we are going to introduce transparent boundary conditions satisfied by the restriction of the solution to $Y^{0}$ (or $Y^{n}$ ). We shall make extensive use of self-similarity in order to design an approximation method for the Dirichlet-Neumann operator entering this transparent condition. This method, which can be used for numerical simulations, is not as simple as the one used for the Poisson problem, because the equation is not invariant by rescaling: as we go toward the finest structures of the ramified domain, diffusion effects dominate and the wave is exponentially dampened. Besides, this is precisely why the approximation of the Dirichlet-Neumann operator will be possible. In this paper, we stay at the continuous level; related numerical methods and simulations are presented in [2].

\subsection{The boundary value problem}

For an integer $n \geq 0$, and for $\sigma \in \mathcal{A}_{n}$, given a real number $k$ and $u \in H^{\frac{1}{2}}\left(\Gamma^{\sigma}\right)$, let us consider the variational problem: find $\hat{u} \in \bar{H}^{1}\left(\Omega^{\sigma}\right)$ such that

$$
\left.\hat{u}\right|_{\Gamma^{\sigma}}=u \quad \text { and } \quad \forall v \in \mathcal{V}\left(\Omega^{\sigma}\right), \quad \int_{\Omega^{\sigma}} \nabla \hat{u} \cdot \nabla v-k \int_{\Omega^{\sigma}} \hat{u} v=0 .
$$

If it exists, $\hat{u}$ is a weak solution to the Helmholtz equation $\Delta \hat{u}+k \hat{u}=0$ in $\Omega^{\sigma}$.

Let us define the operator $L_{k}^{\sigma}$ :

$$
L_{k}^{\sigma}: \mathcal{V}\left(\Omega^{\sigma}\right) \mapsto\left(\mathcal{V}\left(\Omega^{\sigma}\right)\right)^{\prime}, \quad\left\langle L_{k}^{\sigma} w, v\right\rangle=\int_{\Omega^{\sigma}} \nabla w \cdot \nabla v-k \int_{\Omega^{\sigma}} w v .
$$

A scaling argument yields that, for all $\sigma \in \mathcal{A}_{n}, v, w \in \mathcal{V}\left(\Omega^{\sigma}\right)$,

$$
\left\langle L_{k}^{\sigma} w, v\right\rangle=\left\langle L_{\frac{k}{4^{n}}}^{0}\left(w \circ \mathcal{M}_{\sigma}\left(F_{1}, F_{2}\right)\right), v \circ \mathcal{M}_{\sigma}\left(F_{1}, F_{2}\right)\right\rangle .
$$

Let us call $\left(\operatorname{ker}\left(L_{k}^{\sigma}\right)\right)^{\circ}$ the closed subspace of $H^{\frac{1}{2}}\left(\Gamma^{\sigma}\right)$ :

$$
\left(\operatorname{ker}\left(L_{k}^{\sigma}\right)\right)^{\circ}=\left\{u \in H^{\frac{1}{2}}\left(\Gamma^{\sigma}\right) \text { s.t. } \mid \begin{array}{l}
\forall \tilde{u} \in H^{1}\left(\Omega^{\sigma}\right) \text { with }\left.\tilde{u}\right|_{\Gamma^{\sigma}}=u, \\
\int_{\Omega^{\sigma}} \nabla \tilde{u} \cdot \nabla v-k \tilde{u} v=0, \quad \forall v \in \operatorname{ker}\left(L_{k}^{\sigma}\right)
\end{array}\right\} .
$$

From the geometrical self-similarity, it can be verified that for all $\sigma \in \mathcal{A}_{n}$,

$$
\left(\operatorname{ker}\left(L_{\frac{k}{4^{n}}}^{0}\right)\right)^{\circ}=\left\{u \circ \mathcal{M}_{\sigma}\left(F_{1}, F_{2}\right), u \in\left(\operatorname{ker}\left(L_{k}^{\sigma}\right)\right)^{\circ}\right\} .
$$

Theorem 2, the self-similarity of $\Omega^{0}$ and Fredholm's alternative lead to

Proposition 5. For all $n \in \mathbb{N}$, there exists a countable set $S p^{n}=\left\{\lambda_{p}, p \in \mathbb{N}\right\}$ of positive numbers, with $\lambda_{p} \leq \lambda_{p+1}$ and $\lim _{p \rightarrow \infty} \lambda_{p}=+\infty$ such that for $\sigma \in \mathcal{A}_{n}$,

- for $k \in \mathbb{R} \backslash S p^{n}$, the operator $L_{k}^{\sigma}$ is one to one, with a bounded inverse;

- for all $k \in S p^{n}, \operatorname{ker}\left(L_{k}^{\sigma}\right)$ has a positive and finite dimension.

One can obtain a Hilbertian basis of $\mathcal{V}\left(\Omega^{\sigma}\right)$ by assembling bases of $\operatorname{ker}\left(L_{k}^{\sigma}\right), k \in S p^{n}$.

We have

$$
S p^{n}=4^{n} S p^{0} .
$$


For $u \in\left(\operatorname{ker}\left(L_{k}^{\sigma}\right)\right)^{\circ}$, (see (59)), there exists $\hat{u} \in H^{1}\left(\Omega^{\sigma}\right)$ satisfying (56), and $\hat{u}$ is unique up to the addition of functions in $\operatorname{ker}\left(L_{k}^{\sigma}\right)$. Problem (56) defines an injective bounded operator $\mathcal{H}_{k}^{\sigma}$

$$
\mathcal{H}_{k}^{\sigma}:\left(\operatorname{ker}\left(L_{k}^{\sigma}\right)\right)^{\circ} \rightarrow H^{1}\left(\Omega^{\sigma}\right) / \operatorname{ker}\left(L_{k}^{\sigma}\right), \quad \mathcal{H}_{k}^{\sigma}(u)=\hat{u} \bmod \left(\operatorname{ker}\left(\mathrm{L}_{\mathrm{k}}^{\sigma}\right)\right) .
$$

Proof. Let us focus first on the case $n=0$. The compact imbedding of $H^{1}\left(\Omega^{0}\right)$ in $L^{2}\left(\Omega^{0}\right)$, stated in Theorem 2, implies the existence of $S p^{0}$ with the properties stated above. We have seen in Section 4 that $0 \notin S p^{0}$. Then, for $n>0$, identity (58) yields that $S p^{n}$ given by (61) has the properties stated above. The last statement of Proposition 5 is a consequence of Fredholm's alternative.

Remark 12. In relation with Proposition 5, we know from (61) that for any $k \in \mathbb{R}$, there exists a nonnegative integer $N(k)=\min \left\{n \in \mathbb{N}\right.$ such that $\forall p \geq n, \forall \sigma \in \mathcal{A}_{p}, L_{k}^{\sigma}$ is coercive on $\left.\mathcal{V}\left(\Omega^{\sigma}\right)\right\}$. We have $N(k)=0$ if $k \leq 0$ and $N(k) \sim \log (k)$ as $k \rightarrow+\infty$.

From the geometrical self-similarity, it can be verified that for all $\sigma \in \mathcal{A}_{n}$,

$$
\mathcal{H}_{k}^{\sigma} \circ \mathcal{M}_{\sigma}=\mathcal{H}_{\frac{k}{4^{n}}}^{0} .
$$

Remark 13. One can prove the analogue of Lemma 4: there exist two positive constants $k_{0}$ and $\mu<1$ such that, for all $k<k_{0}, \operatorname{ker}\left(L_{k}^{0}\right)=\{0\}$ and for all $u \in H^{\frac{1}{2}}\left(\Gamma^{0}\right),\left\|\mathcal{H}_{k}^{0}(u)\right\|_{H^{1}\left(\Omega^{1}\right)} \leq \mu\left\|\mathcal{H}_{k}^{0}(u)\right\|_{H^{1}\left(\Omega^{0}\right)}$. From this, (63) and Remark 12, we have the analogue of Theorem 6: for all $u \in\left(\operatorname{ker}\left(L_{k}^{0}\right)\right)^{\circ},\left\|\mathcal{H}_{k}^{0}(u)\right\|_{H^{1}\left(\Omega^{p}\right)}$ decays exponentially with $p$ as $p \rightarrow \infty$.

\subsection{The Dirichlet-Neumann operators and transparent boundary conditions}

For $\sigma \in \mathcal{A}_{n}, n \geq 0$, the Dirichlet-Neumann operator $T_{k}^{\sigma}:\left(\operatorname{ker}\left(L_{k}^{\sigma}\right)\right)^{\circ} \mapsto\left(\left(\operatorname{ker}\left(L_{k}^{\sigma}\right)\right)^{\circ}\right)^{\prime}$ is defined by: $\forall u, v \in\left(\operatorname{ker}\left(L_{k}^{\sigma}\right)\right)^{\circ}$,

$$
\left\langle T_{k}^{\sigma} u, v\right\rangle=\int_{\Omega^{\sigma}} \nabla \mathcal{H}_{k}^{\sigma}(u) \cdot \nabla \tilde{v}-k \int_{\Omega^{\sigma}} \mathcal{H}_{k}^{\sigma}(u) \tilde{v},
$$

for any function $\tilde{v} \in H^{1}\left(\Omega^{\sigma}\right)$ such that $\left.\tilde{v}\right|_{\Gamma^{\sigma}}=v$, where, when $k \in S p^{n}, \mathcal{H}_{k}^{\sigma}(u)$ stands for any function in the class $\mathcal{H}_{k}^{\sigma}(u)$. We have that, for all $u, v \in\left(\operatorname{ker}\left(L_{k}^{\sigma}\right)\right)^{\circ}$,

$$
\left\langle T_{k}^{\sigma} u, v\right\rangle=\left\langle T_{\frac{k}{4^{n}}}^{0}\left(u \circ \mathcal{M}_{\sigma}\left(F_{1}, F_{2}\right)\right), v \circ \mathcal{M}_{\sigma}\left(F_{1}, F_{2}\right)\right\rangle .
$$

For $k \notin S p^{n}, T_{k}^{\sigma}$ is a bounded self-adjoint operator from $H^{\frac{1}{2}}\left(\Gamma^{\sigma}\right)$ to its dual.

Lemma 7. The operator $T_{k}^{\sigma}$ is a perturbation of a bounded self-adjoint coercive operator from $\left(\operatorname{ker}\left(L_{k}^{\sigma}\right)\right)^{\circ}$ to $\left(\left(\operatorname{ker}\left(L_{k}^{\sigma}\right)\right)^{\circ}\right)^{\prime}$ by a compact operator.

Proof. For a positive constant $\alpha$, one may write, for any function $\tilde{v} \in H^{1}\left(\Omega^{\sigma}\right)$ such that $\left.\tilde{v}\right|_{\Gamma^{\sigma}}=v$,

$$
\left\langle T_{k}^{\sigma} u, v\right\rangle=\int_{\Omega^{\sigma}} \nabla \mathcal{H}_{k}^{\sigma}(u) \cdot \nabla \mathcal{H}_{k}^{\sigma}(u)+\alpha \int_{\Gamma^{\sigma}} u v-k \int_{\Omega^{\sigma}} \mathcal{H}_{k}^{\sigma}(u) \mathcal{H}_{k}^{\sigma}(v)-\alpha \int_{\Gamma^{\sigma}} u v .
$$

But from (13), we know that for $\alpha$ large enough, the operator $\widehat{T}_{k}^{\sigma}$ :

$$
\left\langle\widehat{T}_{k}^{\sigma} u, v\right\rangle=\int_{\Omega^{\sigma}} \nabla \mathcal{H}_{k}^{\sigma}(u) \cdot \nabla \mathcal{H}_{k}^{\sigma}(v)+\alpha \int_{\Gamma^{\sigma}} u v-k \int_{\Omega^{\sigma}} \mathcal{H}_{k}^{\sigma}(u) \mathcal{H}_{k}^{\sigma}(v)
$$

is coercive, whereas $T_{k}^{\sigma}-\widehat{T}_{k}^{\sigma}$ is clearly compact. 
Lemma 8. For all $u \in\left(\operatorname{ker}\left(L_{k}^{0}\right)\right)^{\circ}$, the restriction $\hat{u}$ to $Y^{n-1}$ of any function in the class $\mathcal{H}_{k}^{0}(u)$ is a solution to the following boundary value problem: for all $\sigma \in \mathcal{A}_{n}$,

$$
\left.\hat{u}\right|_{\Gamma^{\sigma}} \in\left(\operatorname{ker}\left(L_{k}^{\sigma}\right)\right)^{\circ}
$$

and

$$
\left.\hat{u}\right|_{\Gamma^{0}}=u, \quad \text { and } \quad \int_{Y^{n-1}} \nabla \hat{u} \cdot \nabla v-k \int_{Y^{n-1}} \hat{u} v+\sum_{\sigma \in \mathcal{A}_{n}}\left\langle\left. T_{k}^{\sigma} \hat{u}\right|_{\Gamma^{\sigma}},\left.v\right|_{\Gamma^{\sigma}}\right\rangle=0
$$

for all $v \in \mathcal{V}\left(Y^{n-1}\right)$, such that for all $\sigma \in \mathcal{A}_{n},\left.v\right|_{\Gamma^{\sigma}} \in\left(\operatorname{ker}\left(L_{k}^{\sigma}\right)\right)^{\circ}$. A solution to (67) and (68) can be extended to a solution to (56) in a unique manner. Problem (67), (68) has a unique solution up to the addition of restrictions of functions of $\operatorname{ker}\left(L_{k}^{0}\right)$ to $Y^{n-1}$. Furthermore, $\forall v \in H^{1}\left(Y^{n-1}\right)$, such that $\left.v\right|_{\Gamma^{0}} \in\left(\operatorname{ker}\left(L_{k}^{0}\right)\right)^{\circ}$, and for all $\sigma \in \mathcal{A}_{n},\left.v\right|_{\Gamma^{\sigma}} \in\left(\operatorname{ker}\left(L_{k}^{\sigma}\right)\right)^{\circ}$,

$$
\begin{aligned}
\left\langle T_{k}^{0} u,\left.v\right|_{\Gamma^{0}}\right\rangle & =\int_{Y^{n-1}} \nabla \hat{u} \cdot \nabla v-k \int_{Y^{n-1}} \hat{u} v+\sum_{\sigma \in \mathcal{A}_{n}}\left\langle\left. T_{k}^{\sigma} \hat{u}\right|_{\Gamma^{\sigma}},\left.v\right|_{\Gamma^{\sigma}}\right\rangle \\
& =\int_{Y^{n-1}} \nabla \hat{u} \cdot \nabla v-k \int_{Y^{n-1}} \hat{u} v+\sum_{\sigma \in \mathcal{A}_{n}}\left\langle T_{\frac{k}{4^{n}}}^{0}\left(\left.\hat{u}\right|_{\Gamma^{\sigma}} \circ \mathcal{M}_{\sigma}\left(F_{1}, F_{2}\right)\right),\left.v\right|_{\Gamma^{\sigma}} \circ \mathcal{M}_{\sigma}\left(F_{1}, F_{2}\right)\right\rangle .
\end{aligned}
$$

Proof. We skip the proof that the restriction of a function in $\mathcal{H}_{k}^{0}(u)$ to $Y^{n-1}$ satisfies (67) and (68), since this is easily seen. We aim at proving that each solution to (67) and (68) can be extended in a unique manner to a solution of the original problem (56). This is clear if $\frac{k}{4^{n}} \notin S p^{0}$. Thus, we consider the case when $\frac{k}{4^{n}} \in S p^{0}$.

The subspace $\operatorname{ker}\left(L_{k}^{\sigma}\right)$ is finite dimensional. Furthermore, we can apply Holmgren's unique continuation theorem, see [21]: if $v \in \operatorname{ker}\left(L_{k}^{\sigma}\right)$ and if the normal derivative of $v$ on $\Gamma^{\sigma}$ is zero, then $v=0$. Note that $\left.\frac{\partial v}{\partial n}\right|_{\Gamma^{\sigma}}=0$ if and only if the operator from $H^{\frac{1}{2}}\left(\Gamma^{\sigma}\right)$ to its dual: $w \mapsto \int_{\Omega^{\sigma}} \nabla \tilde{w} \cdot \nabla v-k \int_{\Omega^{\sigma}} \tilde{w} v$, where $\tilde{w}$ is any lifting of $w$ in $H^{1}\left(\Omega^{\sigma}\right)$, is zero.

If the dimension of $\operatorname{ker}\left(L_{k}^{\sigma}\right)$ is $d>0$, let $\left(\phi_{\sigma, i}\right)_{i=1, \ldots, d}$ be a basis of $\operatorname{ker}\left(L_{k}^{\sigma}\right)$. From the previous unique continuation result, we see that $\left.\frac{\partial \phi_{\sigma, i}}{\partial n}\right|_{\Gamma^{\sigma}}, i=1, \ldots, d$ are linearly independent. This implies that $\left\{\left(\left\langle\left.\frac{\partial \phi_{\sigma, i}}{\partial n}\right|_{\Gamma^{\sigma}}, \psi\right\rangle\right)_{i=1, \ldots, d}\right.$, $\left.\psi \in H^{\frac{1}{2}}\left(\Gamma^{\sigma}\right)\right\}=\mathbb{R}^{d}$. Therefore, there exists a family $\left(\psi_{\sigma, i}\right)_{i=1, \ldots, d}$ of linearly independent functions in $H^{\frac{1}{2}}\left(\Gamma^{\sigma}\right)$ such that $\int_{\Omega^{\sigma}} \nabla \tilde{\psi}_{\sigma, i} \cdot \nabla \phi_{\sigma, j}-k \int_{\Omega^{\sigma}} \tilde{\psi}_{\sigma, i} \phi_{\sigma, j}=\delta_{i, j}$, where $\tilde{\psi}_{\sigma, i}$ is an arbitrarily chosen function in $\mathcal{V}\left(\Omega^{0}\right)$ such that $\left.\tilde{\psi}_{\sigma, i}\right|_{\Gamma^{\sigma}}=\psi_{\sigma, i}$ and $\left.\tilde{\psi}_{\sigma, i}\right|_{\Omega^{\sigma^{\prime}}}=0$ for each $\sigma^{\prime} \in \mathcal{A}_{n}, \sigma^{\prime} \neq \sigma$. We have

$$
\mathcal{V}\left(\Omega^{0}\right)=\left\{v \in \mathcal{V}\left(\Omega^{0}\right),\left.v\right|_{\Gamma^{\sigma}} \in\left(\operatorname{ker}\left(L_{k}^{\sigma}\right)\right)^{\circ}, \forall \sigma \in \mathcal{A}_{n}\right\} \oplus\left(\bigoplus_{\sigma \in \mathcal{A}_{n}} \operatorname{Span}\left(\tilde{\psi}_{\sigma, j}, j=1, \ldots, d\right)\right) .
$$

Let $\hat{u}$ be a solution to (67) and (68): in order to extend it to a solution of (56), we have, for each $\sigma \in \mathcal{A}_{n}$, to choose the extension in $\mathcal{H}_{k}^{\sigma}\left(\left.\hat{u}\right|_{\Gamma^{\sigma}}\right)$, which is well defined since $\left.\hat{u}\right|_{\Gamma^{\sigma}} \in\left(\operatorname{ker}\left(L_{k}^{\sigma}\right)\right)^{\circ}$. With any such choice, the extended function belongs to $H^{1}\left(\Omega^{0}\right)$ and satisfies the Helmholtz equation in $Y^{n-1}$ and $\Omega^{\sigma}, \sigma \in \mathcal{A}_{n}$. But, for the extension to satisfy (56), its normal derivative must also be continuous across $\Gamma^{\sigma}, \sigma \in \mathcal{A}_{n}$. It can easily be seen that for each $\sigma \in \mathcal{A}_{n}$, there exists a unique function $\hat{u}_{\sigma} \in \mathcal{H}_{k}^{\sigma}\left(\left.\hat{u}\right|_{\Gamma^{\sigma}}\right)$, such that calling $\tilde{u}$ the extension of $\hat{u}$ by $\hat{u}_{\sigma}$ in $\Omega^{\sigma}, \forall \sigma \in \mathcal{A}_{n}$, we have $\forall \sigma \in \mathcal{A}_{n}, \forall i=1, \ldots, d, \int_{\Omega^{0}} \nabla \tilde{u} \cdot \nabla \tilde{\psi}_{\sigma, i}-k \int_{\Omega^{0}} \tilde{u} \tilde{\psi}_{\sigma, i}=0$. From this and from (67), (68) and (70), we deduce that $\tilde{u}$ is a solution to (56).

The last two assertions of the Lemma follow easily. 
We see from (65) (68) that, if the nonlocal operator $T_{\frac{k}{4^{n}}}^{0}$ is known (which implies that $\left(\operatorname{ker}\left(L_{\frac{k}{4^{n}}}^{0}\right)^{\circ}\right.$ is known), then the restriction of $\mathcal{H}_{k}^{0}(u)$ to $Y^{n-1}, n \geq 1$, is characterized as the solution of a boundary value problem in $Y^{n-1}$, with a boundary condition involving $T_{\frac{k}{4^{n}}}^{0}$. Moreover, in the most frequent case when $\frac{k}{4^{p}} \notin S p^{0}$, $\forall p=0, \ldots, n$, and if the operators $T_{\frac{k}{\mu^{p}}}^{0}, p=1, \ldots, n$ are known (or accurately approximated), then solving (68) is equivalent to successively solving $1+2+\cdots+2^{n-1}$ boundary value problems in $Y^{0}$ as follows:

\section{Algorithm 2.}

- Loop: for $p=0$ to $n-1$,

- Loop : for $\sigma \in \mathcal{A}_{p}$, (at this point, $\left.\hat{u}\right|_{\Gamma^{\sigma}}$ is known)

* Solve the boundary value problem in $Y^{0}$ : find $w \in H^{1}\left(Y^{0}\right)$ such that $\left.w\right|_{\Gamma^{0}}=\left.\hat{u}\right|_{\Gamma^{\sigma}} \circ$ $\mathcal{M}_{\sigma}\left(F_{1}, F_{2}\right)$ and $\forall v \in \mathcal{V}\left(Y^{0}\right)$

$$
\begin{aligned}
& \int_{Y^{0}}\left(\nabla w \cdot \nabla v-\frac{k}{4^{p}} w v\right)+\left\langle T_{\frac{k}{4^{p+1}}}^{0}\left(\left.w\right|_{F_{1}\left(\Gamma^{0}\right)} \circ F_{1}\right),\left.v\right|_{F_{1}\left(\Gamma^{0}\right)} \circ F_{1}\right\rangle \\
&+\left\langle T_{\frac{k}{4^{p+1}}}^{0}\left(\left.w\right|_{F_{2}\left(\Gamma^{0}\right)} \circ F_{2}\right),\left.v\right|_{F_{2}\left(\Gamma^{0}\right)} \circ F_{2}\right\rangle=0 . \\
& * \text { Set }\left.\hat{u}\right|_{\mathcal{M}_{\sigma}\left(F_{1}, F_{2}\right)\left(Y^{0}\right)}=w \circ\left(\mathcal{M}_{\sigma}\left(F_{1}, F_{2}\right)\right)^{-1} .
\end{aligned}
$$

In the general case, if $\frac{k}{4^{p}} \in S p^{0}$, for some $p, 1 \leq p<n$, then, as in the proof of Lemma 8, additional finite dimensional linear systems must be solved at the step $p$ of Algorithm 2, in order to enforce the continuity of the normal derivative of $\hat{u}$ at the interfaces $\Gamma^{\sigma}, \sigma \in \mathcal{A}_{p}$.

This method can be transposed at the discrete level (see [2] for a related numerical method and simulations).

\subsection{Approximations of the Dirichlet-Neumann operators}

\subsubsection{Orientation}

There remains the need to compute the operators $T_{\frac{k}{4 p}}^{0}, 0 \leq p \leq n$. The equations (65), (68), (69) can be seen as a backward induction formula with respect to $p$, in order to compute $T_{k}^{0}$. The backward character of the induction makes the exact construction of $T_{k}^{0}$ impossible. Yet, observing that $\lim _{n \rightarrow \infty} T_{\frac{k}{4}}^{0}=T^{0}\left(T^{0}\right.$ is the Dirichlet-Neumann operator for Laplace's equation, see (43)) enables the initialization of the induction by approximating $T_{\frac{k}{4^{n}}}^{0}$ by $T^{0}$, for $n$ large enough. The goal of what follows is to carry out this program in detail.

\subsubsection{An induction formula to approximate the Dirichlet-Neumann operators}

For $\sigma \in \mathcal{A}_{n}, n \geq 0$, and $r \in \mathbb{N}, r \geq n$, let us introduce the operators $L_{k}^{\sigma, r}$

$$
L_{k}^{\sigma, r}: \mathcal{V}\left(\Omega^{\sigma}\right) \mapsto\left(\mathcal{V}\left(\Omega^{\sigma}\right)\right)^{\prime}, \quad\left\langle L_{k}^{\sigma, r} u, v\right\rangle=\int_{\Omega^{\sigma}} \nabla u \cdot \nabla v-k \int_{Y^{r-1} \cap \Omega^{\sigma}} u v
$$

agreeing that $Y^{-1}=\emptyset$. Note that for $u \in H^{\frac{1}{2}}\left(\Gamma^{\sigma}\right)$, a function $\hat{u} \in H^{1}\left(\Omega^{\sigma}\right)$ such that

$$
\left.\hat{u}\right|_{\Gamma^{\sigma}}=u \quad \text { and } \quad \forall v \in \mathcal{V}\left(\Omega^{\sigma}\right), \quad \int_{\Omega^{\sigma}} \nabla \hat{u} \cdot \nabla v-k \int_{Y^{r-1} \cap \Omega^{\sigma}} \hat{u} v=0,
$$

is a weak solution to the Helmholtz equation $\Delta \hat{u}+k 1_{Y^{r-1} \cap \Omega^{\sigma}} \hat{u}=0$ in $\Omega^{\sigma}$. 
Let us call $\left(\operatorname{ker}\left(L_{k}^{\sigma, r}\right)\right)^{\circ}$ the closed subspace of $H^{\frac{1}{2}}\left(\Gamma^{\sigma}\right)$ :

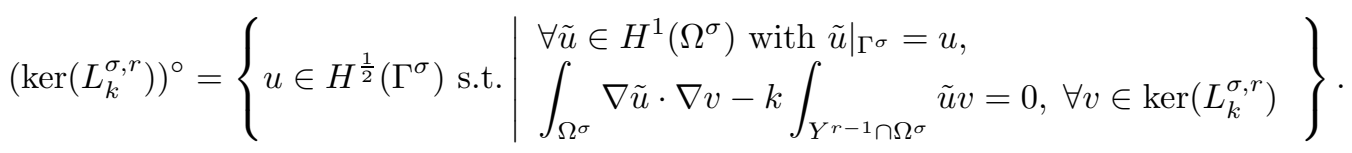

We have the analogue of Proposition 5:

Proposition 6. For all $n, r \in \mathbb{N}$ with $r \geq n$, there exists a countable set $S p^{n, r}=\left\{\lambda_{q}, q \in \mathbb{N}\right\}$ of positive numbers, with $\lambda_{q} \leq \lambda_{q+1}$ and $\lim _{q \rightarrow \infty} \lambda_{q}=+\infty$ such that, for all $\sigma \in \mathcal{A}_{n}$,

- for all $k \in \mathbb{R} \backslash S p^{n, r}$, the operator $L_{k}^{\sigma, r}$ is one to one, with a bounded inverse;

- for all $k \in S p^{n, r}, \operatorname{ker}\left(L_{k}^{\sigma, r}\right)$ has a positive and finite dimension.

We have

$$
S p^{n, r}=4^{n} S p^{0, r-n} .
$$

If $u \in\left(\operatorname{ker}\left(L_{k}^{\sigma, r}\right)\right)^{\circ}$, then there exists $\hat{u} \in H^{1}\left(\Omega^{\sigma}\right)$ satisfying (71) and $\hat{u}$ is unique up to functions in $\operatorname{ker}\left(L_{k}^{\sigma, r}\right)$. Problem (71) defines an injective bounded operator $\mathcal{H}_{k}^{\sigma, r}$

$$
\mathcal{H}_{k}^{\sigma, r}:\left(\operatorname{ker}\left(L_{k}^{\sigma, r}\right)\right)^{\circ} \rightarrow H^{1}\left(\Omega^{\sigma}\right) / \operatorname{ker}\left(L_{k}^{\sigma, r}\right), \quad \mathcal{H}_{k}^{\sigma, r}(u)=\hat{u} \bmod \left(\operatorname{ker}\left(\mathrm{L}_{\mathrm{k}}^{\sigma, \mathrm{r}}\right)\right) .
$$

The Dirichlet-Neumann operator $T_{k}^{\sigma, r}:\left(\operatorname{ker}\left(L_{k}^{\sigma, r}\right)\right)^{\circ} \mapsto\left(\left(\operatorname{ker}\left(L_{k}^{\sigma, r}\right)\right)^{\circ}\right)^{\prime}$ is defined by: $\forall u, v \in\left(\operatorname{ker}\left(L_{k}^{\sigma, r}\right)\right)^{\circ}$,

$$
\left\langle T_{k}^{\sigma, r} u, v\right\rangle=\int_{\Omega^{\sigma}} \nabla \mathcal{H}_{k}^{\sigma, r}(u) \cdot \nabla \tilde{v}-k \int_{Y^{r-1} \cap \Omega^{\sigma}} \mathcal{H}_{k}^{\sigma, r}(u) \tilde{v},
$$

for any function $\tilde{v} \in H^{1}\left(\Omega^{\sigma}\right)$ such that $\left.\tilde{v}\right|_{\Gamma^{\sigma}}=v$, and where $\mathcal{H}_{k}^{\sigma, r}(u)$ stands for any function in the class $\mathcal{H}_{k}^{\sigma, r}(u)$ if $k \in S p^{n, r}$.

The analogues of Lemmas 7 and 8 are summarized in the following result:

Lemma 9. The operator $T_{k}^{\sigma, r}$ is the perturbation of a bounded and coercive self-adjoint operator from $\left(\operatorname{ker}\left(L_{k}^{\sigma, r}\right)\right)^{\circ}$ to $\left(\left(\operatorname{ker}\left(L_{k}^{\sigma, r}\right)\right)^{\circ}\right)^{\prime}$ by a compact operator.

For all $n, r \in \mathbb{N}$, with $n \leq r$, for all $\sigma \in \mathcal{A}_{n}$ and $u, v \in\left(\operatorname{ker}\left(L_{k}^{\sigma, r}\right)\right)^{\circ}$, we have $u \circ \mathcal{M}_{\sigma}\left(F_{1}, F_{2}\right), v \circ \mathcal{M}_{\sigma}\left(F_{1}, F_{2}\right) \in$ $\left(\operatorname{ker}\left(L_{\frac{k}{4^{n}}}^{0, r-n}\right)\right)^{\circ}$, and

$$
\left\langle T_{k}^{\sigma, r} u, v\right\rangle=\left\langle T_{\frac{k}{4^{n}}}^{0, r-n}\left(u \circ \mathcal{M}_{\sigma}\left(F_{1}, F_{2}\right)\right), v \circ \mathcal{M}_{\sigma}\left(F_{1}, F_{2}\right)\right\rangle .
$$

If $n \geq 1$, then for all $u \in\left(\operatorname{ker}\left(L_{k}^{0, r}\right)\right)^{\circ}$, the restriction $\hat{u}$ to $Y^{n-1}, n \geq 1$, of any function in the class $\mathcal{H}_{k}^{0, r}(u)$ satisfies the following boundary value problem: for all $\sigma \in \mathcal{A}_{n}$,

$$
\left.\hat{u}\right|_{\Gamma^{\sigma}} \in\left(\operatorname{ker}\left(L_{k}^{\sigma, r}\right)\right)^{\circ}
$$

and

$$
\left.\hat{u}\right|_{\Gamma^{0}}=u, \quad \text { and } \quad \int_{Y^{n-1}} \nabla \hat{u} \cdot \nabla v-k \int_{Y^{n-1}} \hat{u} v+\sum_{\sigma \in \mathcal{A}_{n}}\left\langle T_{k}^{\sigma, r}\left(\left.\hat{u}\right|_{\Gamma^{\sigma}}\right),\left.v\right|_{\Gamma^{\sigma}}\right\rangle=0,
$$

$\forall v \in \mathcal{V}\left(Y^{n-1}\right)$ such that for all $\sigma \in \mathcal{A}_{n},\left.v\right|_{\Gamma^{\sigma}} \in\left(\operatorname{ker}\left(L_{k}^{\sigma, r}\right)\right)^{\circ}\left((78)\right.$ can be written in terms of $T_{\frac{k}{4^{n}}}^{0, r-n}$ thanks to (76)). Problem (77)-(78) has a unique solution up to restrictions of functions of $\operatorname{ker}\left(L_{k}^{0, r}\right)$ to $Y^{n-1}$. Furthermore, $\forall v \in H^{1}\left(Y^{n-1}\right)$, such that $\left.v\right|_{\Gamma^{0}} \in\left(\operatorname{ker}\left(L_{k}^{0, r}\right)\right)^{\circ}$ and for all $\sigma \in \mathcal{A}_{n},\left.v\right|_{\Gamma^{\sigma}} \in\left(\operatorname{ker}\left(L_{k}^{\sigma, r}\right)\right)^{\circ}$,

$$
\begin{aligned}
& \left\langle T_{k}^{0, r} u,\left.v\right|_{\Gamma^{0}}\right\rangle=\int_{Y^{n-1}} \nabla \hat{u} \cdot \nabla v-k \int_{Y^{n-1}} \hat{u} v+\sum_{\sigma \in \mathcal{A}_{n}}\left\langle\left. T_{k}^{\sigma, r} \hat{u}\right|_{\Gamma^{\sigma}},\left.v\right|_{\Gamma^{\sigma}}\right\rangle \\
& =\int_{Y^{n-1}} \nabla \hat{u} \cdot \nabla v-k \int_{Y^{n-1}} \hat{u} v+\sum_{\sigma \in \mathcal{A}_{n}}\left\langle T_{\frac{k}{4^{n}}}^{0, r-n}\left(\left.\hat{u}\right|_{\Gamma^{\sigma}} \circ \mathcal{M}_{\sigma}\left(F_{1}, F_{2}\right)\right),\left.v\right|_{\Gamma^{\sigma}} \circ \mathcal{M}_{\sigma}\left(F_{1}, F_{2}\right)\right\rangle .
\end{aligned}
$$


From Lemma 9, we see that if $T^{0}$ is available, then one can construct $T_{k}^{0, r}$ by the following induction:

Algorithm 3 (the inductive construction of $\left.T_{k}^{0, r}\right)$. Let us construct the closed subspaces $\mathcal{D}^{(j)}$ of $H^{\frac{1}{2}}\left(\Gamma^{0}\right)$ and the operators $\left(Z^{(j)}\right)_{0 \leq j \leq r}$ by

- $\mathcal{D}^{(0)}=H^{\frac{1}{2}}\left(\Gamma^{0}\right)$ and $Z^{(0)}=T^{0}$.

- Induction formula (I.F.). Suppose that after $j$ steps, $j<r$, we have constructed the closed subspace $\mathcal{D}^{(j)}$ of $H^{\frac{1}{2}}\left(\Gamma^{0}\right)$ with finite codimension and the operator $Z^{(j)}$, from $\mathcal{D}^{(j)}$ to its dual, such that $Z^{(j)}$ is a perturbation of a coercive self-adjoint operator on $\mathcal{D}^{(j)}$ by a compact operator. We call $W^{(j)}$ the finite dimensional space containing the functions $w \in \mathcal{V}\left(Y^{0}\right)$, such that $\left.w\right|_{\Gamma^{\sigma}} \circ \mathcal{M}_{\sigma}\left(F_{1}, F_{2}\right) \in \mathcal{D}^{(j)}, \forall \sigma \in \mathcal{A}_{1}$, and

$$
\begin{aligned}
& \int_{Y^{0}} \nabla w \cdot \nabla v-\frac{k}{4^{r-j-1}} \int_{Y^{0}} w v+\sum_{\sigma \in \mathcal{A}_{1}}\left\langle Z^{(j)}\left(\left.w\right|_{\Gamma^{\sigma}} \circ \mathcal{M}_{\sigma}\left(F_{1}, F_{2}\right)\right),\left.v\right|_{\Gamma^{\sigma}} \circ \mathcal{M}_{\sigma}\left(F_{1}, F_{2}\right)\right\rangle=0, \\
& \forall v \in \mathcal{V}\left(Y^{0}\right), \text { with }\left.v\right|_{\Gamma^{\sigma}} \circ \mathcal{M}_{\sigma}\left(F_{1}, F_{2}\right) \in \mathcal{D}^{(j)}, \forall \sigma \in \mathcal{A}_{1} .
\end{aligned}
$$

We call $\mathcal{D}^{(j+1)}$ the closed subspace of $H^{\frac{1}{2}}\left(\Gamma^{0}\right)$ containing the functions $v$ such that $\forall w \in W^{(j)}$,

$$
\int_{Y^{0}} \nabla w \cdot \nabla \tilde{v}-\frac{k}{4^{r-j-1}} \int_{Y^{0}} w \tilde{v}+\sum_{\sigma \in \mathcal{A}_{1}}\left\langle Z^{(j)}\left(\left.w\right|_{\Gamma^{\sigma}} \circ \mathcal{M}_{\sigma}\left(F_{1}, F_{2}\right)\right),\left.\tilde{v}\right|_{\Gamma^{\sigma}} \circ \mathcal{M}_{\sigma}\left(F_{1}, F_{2}\right)\right\rangle=0
$$

$\forall \tilde{v} \in H^{1}\left(Y^{0}\right)$, with $\left.\tilde{v}\right|_{\Gamma^{\sigma}} \circ \mathcal{M}_{\sigma}\left(F_{1}, F_{2}\right) \in \mathcal{D}^{(j)}, \forall \sigma \in \mathcal{A}_{1}$, and $\left.\tilde{v}\right|_{\Gamma^{0}}=v$.

Then, from Fredholm's alternative, we know that the problem: find $\hat{u} \in H^{1}\left(Y^{0}\right)$ such that $\left.\hat{u}\right|_{\Gamma^{0}}=u,\left.\hat{u}\right|_{\Gamma^{\sigma}} \circ$ $\mathcal{M}_{\sigma}\left(F_{1}, F_{2}\right) \in \mathcal{D}^{(j)}, \forall \sigma \in \mathcal{A}_{1}$, and

$$
\begin{aligned}
& \int_{Y^{0}} \nabla \hat{u} \cdot \nabla v-\frac{k}{4^{r-j-1}} \int_{Y^{0}} \hat{u} v+\sum_{\sigma \in \mathcal{A}_{1}}\left\langle Z^{(j)}\left(\left.\hat{u}\right|_{\Gamma^{\sigma}} \circ \mathcal{M}_{\sigma}\left(F_{1}, F_{2}\right)\right),\left.v\right|_{\Gamma^{\sigma}} \circ \mathcal{M}_{\sigma}\left(F_{1}, F_{2}\right)\right\rangle=0 \\
& \forall v \in \mathcal{V}\left(Y^{0}\right) \text { such that } \forall \sigma \in \mathcal{A}_{1},\left.v\right|_{\Gamma^{\sigma}} \circ \mathcal{M}_{\sigma}\left(F_{1}, F_{2}\right) \in \mathcal{D}^{(j)}
\end{aligned}
$$

has a solution if $u \in \mathcal{D}^{(j+1)}$, which is unique up to functions in $W^{(j)}$. Then we can define the operator $Z^{(j+1)}$ from $\mathcal{D}^{(j+1)}$ to its dual, by: $\forall u, v \in \mathcal{D}^{(j+1)}$,

$$
\begin{aligned}
& \left\langle Z^{(j+1)} u, v\right\rangle=\int_{Y^{0}} \nabla \hat{u} \cdot \nabla \tilde{v}-\frac{k}{4^{r-j-1}} \int_{Y^{0}} \hat{u} \tilde{v}+\sum_{\sigma \in \mathcal{A}_{1}}\left\langle Z^{(j)}\left(\left.\hat{u}\right|_{\Gamma^{\sigma}} \circ \mathcal{M}_{\sigma}\left(F_{1}, F_{2}\right)\right),\left.\tilde{v}\right|_{\Gamma^{\sigma}} \circ \mathcal{M}_{\sigma}\left(F_{1}, F_{2}\right)\right\rangle, \\
& \forall \tilde{v} \in H^{1}\left(Y^{0}\right), \text { with }\left.\tilde{v}\right|_{\Gamma^{\sigma}} \circ \mathcal{M}_{\sigma}\left(F_{1}, F_{2}\right) \in \mathcal{D}^{(j)}, \forall \sigma \in \mathcal{A}_{1}, \text { and }\left.\tilde{v}\right|_{\Gamma^{0}}=v,
\end{aligned}
$$

where $\hat{u}$ is a solution to (80). It is clear that $Z^{(j+1)}$ has the same properties as $Z^{(j)}$.

Proposition 7. The operators constructed by Algorithm 3 satisfy: for $j \leq r$,

$$
Z^{(j)}=T_{\frac{k}{4^{r-j}}}^{0, j} \quad \text { and } \quad \mathcal{D}^{(j)}=\left(\operatorname{ker}\left(\frac{L_{k}^{0, j}}{4^{r-j}}\right)\right)^{\circ}
$$

Proof. By induction.

Remark 14. In fact, for $k$ belonging to a dense subset in $\mathbb{R}$, the domains $\mathcal{D}^{j}, 0 \leq j \leq r$ all coincide with $H^{\frac{1}{2}}\left(\Gamma^{0}\right)$. Proposition 7 says that $T_{k}^{0, r}$ can be constructed inductively, departing from $T^{0}$. 
Finally, the following result says that the original Dirichlet-Neumann operator $T_{k}^{\sigma}, \sigma \in \mathcal{A}_{n}$, can be approximated by the modified operator $T_{k}^{\sigma, r}$, at least when $k \notin S p^{n}$ :

Theorem 8. For $\sigma \in \mathcal{A}_{n}$ and $k \notin S p^{n}$, there exists $R(k, n) \geq n$, such that for all $r \geq R(k, n)$, the operator $L_{k}^{\sigma, r}$ is one to one, and there exists a constant $\mathrm{C}>0$, (depending of $k$ but not of $n$ and $r$ ), such that, for $r \geq R(k, n)$,

$$
\left\|\left(L_{k}^{\sigma, r}\right)^{-1}-\left(L_{k}^{\sigma}\right)^{-1}\right\| \leq \mathrm{C} 2^{-n-r},
$$

and the operator $T_{k}^{\sigma, r}$ is bounded from $H^{\frac{1}{2}}\left(\Gamma^{\sigma}\right)$ to its dual, with

$$
\left\|T_{k}^{\sigma, r}-T_{k}^{\sigma}\right\| \leq \mathrm{C} 2^{-n-r} .
$$

Proof. Since $k \notin S p^{n}, L_{k}^{\sigma}$ is one to one. From (20), we have that $\left\|L_{k}^{\sigma, r}-L_{k}^{\sigma}\right\| \lesssim 2^{-n-r}$ and therefore $\lim _{r \rightarrow \infty}\left\|L_{k}^{\sigma, r}-L_{k}^{\sigma}\right\|=0$. It is a standard matter to deduce (82) and (83) from the last two observations.

\subsubsection{Stability of the approximation of the Dirichlet-Neumann operator}

In practice, $T^{0}$ is not available, and one has to initialize Algorithm 3 by approximations of $T^{0}$. The approximation of $T^{0}$ is constructed by using (53). The following theorem gives an error estimate for the approximation of $T_{k}^{0}$ :

Theorem 9. For all $R \in \mathbb{O}, r, q \in \mathbb{N}$, consider the sequence $Z_{q, r}^{(j)}, 0 \leq j \leq r$ :

- $Z_{q, r}^{(0)}=\mathbb{M}^{q}(R)$

- for $0 \leq j<r, Z_{q, r}^{(j+1)}$ is obtained from $Z_{q, r}^{(j)}$ by the induction (I.F.) above,

where $\mathbb{M}$ has been introduced in (51), (52). Assume that $k \notin S p^{0}$. Then there exist two integers $R(k)$ and $Q(k)$ such that for all $r>R(k)$, for all $q>Q(k) Z_{q, r}^{(r)}$ is a bounded operator from $H^{\frac{1}{2}}\left(\Gamma^{0}\right)$ to its dual, and there exists a constant $\mathrm{C}$ such that for all $r>R(k), q>Q(k)$,

$$
\left\|Z_{q, r}^{(r)}-T_{k}^{0}\right\| \leq \mathrm{C}\left(\rho^{\frac{q}{4}}+2^{-r}\right),
$$

where $0<\rho<1$ is the constant introduced in (40).

Proof. The proof is given in Section 10.

\section{Conclusion}

We have introduced transparent boundary conditions for both the elliptic problem (32) (with $g=0$ ) and for the Helmholtz equation (56). For any $n>0$, they permit finding the solutions in $Y^{n-1}$. Let us summarize how this can be done:

- for problem (32) (with $g=0$ ):

- approximate $T^{0}$ by $\mathbb{M}^{r}(0)$ (see Thm. 7), with a large enough $r$,

- use Algorithm 1 to compute $\left.w\right|_{Y^{n-1}}$;

- for problem (56):

- approximate $T^{0}$ by $\mathbb{M}^{r}(0)$ (see Thm. 7), with a large enough $r$,

- approximate $T_{\frac{k}{4 p}}^{0}, p=0, \ldots, n$ by using Algorithm 3,

- use Algorithm 2 to compute $\left.\hat{u}\right|_{Y^{n-1}}$.

These methods can be transposed at a discrete level by using finite elements with self-similar triangulations (assuming that a unique continuation theorem holds in the discrete case). This is done in [2] for both the Laplace and Helmholtz equations. In [2], a method for computing and normalizing the vibration modes is implemented as well. In [1], the use of transparent boundary conditions in the case when $g \neq 0$ is discussed.

Finally, let us underline the fact that all the algorithms and methods proposed in this paper can be applied for many other self-similar geometries such as the Koch flake, for example. They can also applied in dimension 
three, under the same geometrical assumptions. Although no numerical tests have been done so far in dimension three, one can predict that the complexity of the numerical methods stemming from the theory above remains moderate. The methods above may also be extended to the case when the two mappings $F_{1}$ and $F_{2}$ correspond to two different dilation ratii, as soon as self-similarity is preserved. On the contrary, since they rely heavily on self-similarity, the methods presented here cannot apply to the irregular situations when the dilation ratio changes arbitrarily within one generation.

\section{Proof of Theorem 1}

We proceed by first proving the Poincaré inequality for functions in the space $\mathcal{V}^{q}\left(Y^{N}\right)=\left\{v \in W^{1, q}\left(Y^{N}\right)\right.$ s.t. $\left.\left.v\right|_{\Gamma^{0}}=0\right\}$, with a constant independent of $N$. Since the function space $\left\{v \in \mathcal{C}^{\infty}\left(\overline{Y^{N}}\right)\right.$ s.t. $\left.\left.v\right|_{\Gamma^{0}}=0\right\}$ is dense in $\mathcal{V}^{q}\left(Y^{N}\right)$, it is enough to prove the inequality for functions in that space.

The idea of the proof is to construct explicitly a change of variables which maps $\Omega^{0}$ onto a fractured set contained in the rectangle $(-1,1) \times(0,8)$.

Let us introduce $\mathcal{Q}^{0}=((-1,1) \times(0,2]) \cup((-2,2) \times(2,3)) \cup(((-2,-1) \cup(1,2)) \times\{3\})$. We define first a continuous and piecewise affine change of variables $\chi^{0}$ mapping $\widehat{\mathcal{Q}}^{0}=((-1,1) \times(0,4]) \backslash(\{0\} \times[3,4])$ onto $\mathcal{Q}^{0}$ by

$$
\begin{array}{rlr}
\text { if } x>0, & \chi^{0}(x, t)=\left\{\begin{array}{ll}
(x, t) & \text { for } t \in(0,3-x] \\
(t-3+2 x, 3-x) & \text { for } t \in[3-x, 4-x], \\
(x+1, t-1) & \text { for } t \in[4-x, 4]
\end{array},\right. \\
\text { if } x<0, & \chi^{0}(x, t)=\left(-\chi_{1}^{0}(-x, t), \chi_{2}^{0}(-x, t)\right) & \text { for } t \in(0,4], \\
\chi^{0}(0, t) & =(0, t) \quad \text { for } t \in(0,3) .
\end{array}
$$

It is simple to verify that $\chi^{0}$ is one to one. The set $\widehat{\mathcal{Q}^{0}}$ is fractured in the sense that it does not lie locally on one side of its boundary.

Note also that for each $x \in(-1,1)$, the trajectory $\left\{\chi^{0}(x, t), t \in(0,4]\right\}$ is made of at most three straight lines parallel to the axes, and that for $x \in(0,1), \chi^{0}(x, 4)=x+1$ so $\left\{\chi^{0}(x, 4), x \in(0,1)\right\}=(1,2) \times\{3\}$. Similarly, one can check that $\nabla \chi^{0}$ is piecewise constant and can only take the values

$$
\nabla \chi^{0}=\left(\begin{array}{ll}
1 & 0 \\
0 & 1
\end{array}\right) \quad \text { or } \quad \nabla \chi^{0}=\left(\begin{array}{cc}
2 & 1 \\
-1 & 0
\end{array}\right)
$$

Thus, at the points where $\nabla \chi^{0}$ is defined, $\operatorname{det}\left(\nabla \chi^{0}\right)=1$. Therefore the map $\chi^{0}$ preserves the measure.

Let $H_{1}$ and $H_{2}$ be the affine maps in $\mathbb{R}^{2}$

$$
H_{1}(x)=\left(-\frac{1}{2}+\frac{x_{1}}{2}, 4+\frac{x_{2}}{2}\right), \quad H_{2}(x)=\left(\frac{1}{2}+\frac{x_{1}}{2}, 4+\frac{x_{2}}{2}\right) .
$$

Then we define the one to one mapping

$$
\chi^{N}: \quad \widehat{\mathcal{Q}^{N}}=\cup_{n=0}^{N} \cup_{\sigma \in \mathcal{A}_{n}} \mathcal{M}_{\sigma}\left(H_{1}, H_{2}\right)\left(\widehat{\mathcal{Q}^{0}}\right) \mapsto \cup_{n=0}^{N} \cup_{\sigma \in \mathcal{A}_{n}} \mathcal{M}_{\sigma}\left(F_{1}, F_{2}\right)\left(\mathcal{Q}^{0}\right),
$$

by

$$
\chi^{N}(x)=\left(\mathcal{M}_{\sigma}\left(F_{1}, F_{2}\right) \circ \chi^{0} \circ\left(\mathcal{M}_{\sigma}\left(H_{1}, H_{2}\right)\right)^{-1}\right)(x), \quad \text { if } x \in \mathcal{M}_{\sigma}\left(H_{1}, H_{2}\right)\left(\widehat{\mathcal{Q}^{0}}\right) .
$$

Note that $\widehat{\mathcal{Q}^{N}}$ is contained in the rectangle $(-1,1) \times\left(0,4 s_{N}\right)$ where

$$
s_{N}=\sum_{i=0}^{N} 2^{-i}
$$


and that $\widehat{\mathcal{Q}^{N}}$ has $\sum_{n=0}^{N} 2^{n}$ vertical fractures. It is clear that $\nabla \chi^{N}$ can take only the two values in (85) and that $\chi^{N}$ preserves the measure.

Similarly, we can construct a one to one map $\chi^{\infty}$ from $\omega^{0}=\cup_{n=0}^{\infty} \cup_{\sigma \in \mathcal{A}_{n}} \mathcal{M}_{\sigma}\left(H_{1}, H_{2}\right)\left(\widehat{\mathcal{Q}^{0}}\right)$ to $\Omega^{0}$, which preserves the measure. The sets $\widehat{\mathcal{Q}^{0}}$ and $\omega^{0}$ are displayed in Figure 2 .

Let us define $I_{N}=1+\sum_{i=0}^{N} 2^{i}$ and call $a_{i}, 0 \leq i \leq I_{N}$ the abscissa of the vertical boundaries of $\widehat{\mathcal{Q}^{N}}$, ordered increasingly. Consider a function $u \in C_{0}^{\infty}\left(\overline{Y^{N}}\right)$ such that $\left.u\right|_{\Gamma^{0}}=0$.

$$
\begin{aligned}
\int_{Y^{N}}|u|^{q} & =\int_{\widehat{\mathcal{Q}^{N}}}|u|^{q}\left(\chi^{N}(x, t)\right)=\sum_{i=0}^{I_{N}-1} \int_{a_{i}}^{a_{i+1}} \mathrm{~d} x \int_{0}^{4 s_{N}}|u|^{q}\left(\chi^{N}(x, t)\right) \mathrm{d} t \\
& =\sum_{i=0}^{I_{N}-1} \int_{a_{i}}^{a_{i+1}} \mathrm{~d} x \int_{0}^{4 s_{N}}\left|\int_{0}^{t} \frac{d}{\mathrm{~d} s}\left(u\left(\chi^{N}(x, s)\right)\right) \mathrm{d} s\right|^{q} \mathrm{~d} t \\
& \leq \sum_{i=0}^{I_{N}-1} \int_{a_{i}}^{a_{i+1}} \mathrm{~d} x \int_{0}^{4 s_{N}} \mathrm{~d} t t^{q-1} \int_{0}^{t}\left(\left|\frac{\partial u}{\partial x_{1}}\left(\chi^{N}(x, s)\right) \frac{\partial \chi_{1}^{N}}{\partial t}(x, s)\right|^{q}+\left|\frac{\partial u}{\partial x_{2}}\left(\chi^{N}(x, s)\right) \frac{\partial \chi_{2}^{N}}{\partial t}(x, s)\right|^{q}\right) \mathrm{d} s
\end{aligned}
$$

by Hölder's inequality and because

$$
\frac{\partial \chi_{1}^{N}}{\partial t} \frac{\partial \chi_{2}^{N}}{\partial t}=0
$$

Therefore

$$
\begin{aligned}
\int_{Y^{N}}|u|^{q} & \leq \frac{\left(4 s_{N}\right)^{q}}{q} \sum_{i=0}^{I_{N}-1} \int_{a_{i}}^{a_{i+1}} \mathrm{~d} x \int_{0}^{4 s_{N}}\left(\left|\frac{\partial u}{\partial x_{1}}\left(\chi^{N}(x, s)\right) \frac{\partial \chi_{1}^{N}}{\partial t}(x, s)\right|^{q}+\left|\frac{\partial u}{\partial x_{2}}\left(\chi^{N}(x, s)\right) \frac{\partial \chi_{2}^{N}}{\partial t}(x, s)\right|^{q}\right) \mathrm{d} s \\
& \leq \frac{\left(4 s_{N}\right)^{q}}{q} \sum_{i=0}^{I_{N}-1} \int_{a_{i}}^{a_{i+1}} \mathrm{~d} x \int_{0}^{4 s_{N}}\left|\nabla u\left(\chi^{N}(x, s)\right)\right|^{q} \mathrm{~d} s
\end{aligned}
$$

from (87) and because $\left|\frac{\partial \chi_{1}^{N}}{\partial t}\right| \leq 1$, and $\left|\frac{\partial \chi_{2}^{N}}{\partial t}\right| \leq 1$. Using the fact that $s_{N}<2$ and performing the inverse change of variables, we obtain that

$$
\int_{Y^{N}}|u|^{q} \leq \frac{8^{q}}{q} \int_{Y^{N}}|\nabla u|^{q}
$$

By density, it is clear that (88) holds for $u \in \mathcal{V}^{q}\left(Y^{N}\right)$. Since the constant in (88) does not depend on $N$, we obtain (12) by using Lebesgue's theorem.

\section{Proof of Theorem 3}

\subsection{An intermediate domain}

The construction of the extension operator is done by introducing an intermediate domain $\widetilde{\Omega}^{0}$ displayed on the right side of Figure 3 with full lines, such that $\Omega^{0} \subset \widetilde{\Omega}^{0} \subset \widehat{\Omega}^{0}$, and by composing an extension operator from $\Omega^{0}$ to $\widetilde{\Omega}^{0}$ with an extension operator from $\widetilde{\Omega}^{0}$ to $\widehat{\Omega}^{0}$.

The domain $\widetilde{\Omega}^{0}$ is defined as follows: call $\widetilde{Y}^{0}$ the trapezoidal domain with vertices $\left(-\frac{3}{2}, 0\right),\left(\frac{3}{2}, 0\right),\left(-\frac{5}{2}, 3\right)$ and $\left(\frac{5}{2}, 3\right)$, and $\widetilde{\Omega}^{0}$ the new domain

$$
\widetilde{\Omega}^{0}=\operatorname{Interior}\left(\bigcup_{n \in \mathbb{N}} \bigcup_{\sigma \in \mathcal{A}_{n}} \mathcal{M}_{\sigma}\left(F_{1}, F_{2}\right)\left(\overline{\widetilde{Y}^{0}}\right)\right) .
$$

It is clear that $\Omega^{0} \subset \widetilde{\Omega}^{0}$. 


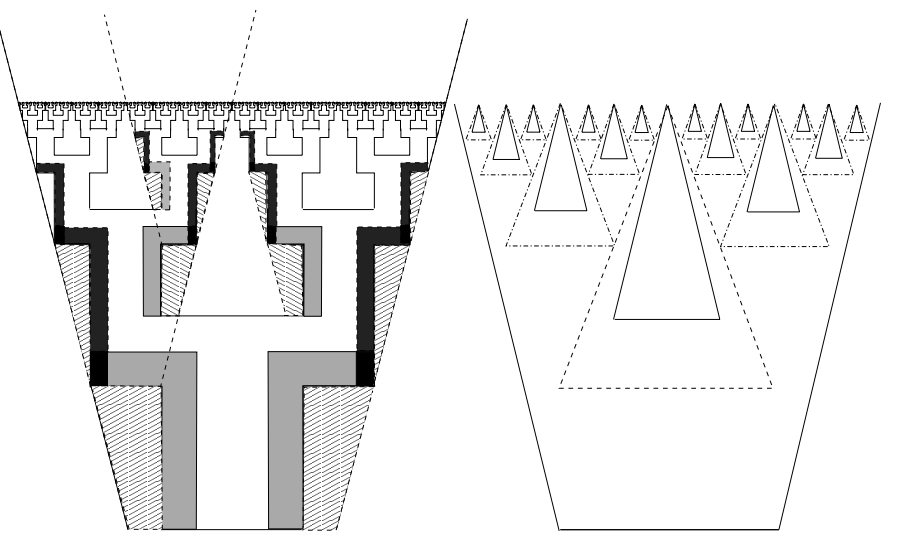

FiguRE 3. The extension is performed in two steps.

Note that $\widetilde{\Omega}^{0}$ is obtained by removing an infinite family of nonoverlapping triangles from $\widehat{\Omega}^{0}$. More precisely, consider the triangle $T$ whose vertices are $(0,6),(1,3)$, and $(-1,3)$ (note that $T \subset \overline{\widehat{\Omega}^{0}} \backslash \widetilde{\Omega}^{0}$ ), which is visible on the right part of Figure 3. We have that

$$
\widetilde{\Omega}^{0}=\widehat{\Omega}^{0} \backslash\left(\bigcup_{n \in \mathbb{N}} \bigcup_{\sigma \in \mathcal{A}_{n}} \mathcal{M}_{\sigma}\left(F_{1}, F_{2}\right) T\right) .
$$

\subsection{Bounded extension from $W^{1, q}\left(\Omega^{0}\right)$ to $W^{1, q}\left(\widetilde{\Omega}^{0}\right), 1 \leq q<\infty$}

Lemma 10. There exists an extension operator $\mathcal{E}$ bounded from $W^{1, q}\left(\Omega^{0}\right)$ to $W^{1, q}\left(\widetilde{\Omega}^{0}\right)$, for all $q, 1 \leq q<\infty$.

Proof. The extension operator $\mathcal{E}$ is constructed in two steps.

First step. Call $D_{L}$ the L-shaped compact set $D_{L}=\operatorname{conv}\left((-1,-2),\left(-\frac{1}{2},-2\right),\left(-\frac{1}{2}, 2\right),(-1,2)\right) \cup \operatorname{conv}\left(\left(-\frac{1}{2}, 2\right)\right.$, $\left.(-2,2),\left(-2, \frac{5}{2}\right),\left(-\frac{1}{2}, \frac{5}{2}\right)\right)$ and $D_{R}$ the image of $D_{L}$ by the symmetry of axis $x_{1}=0$. Call also $T_{L}$ the triangle whose vertices are $(-1,-2),(-1,2)$, and $(-2,2)$ and $T_{R}$ the image of $T_{L}$ by the symmetry of axis $x_{1}=0$.

It is possible to construct an extension operator $\mathcal{E}_{L}$, which maps continuously $W^{1, q}\left(D_{L}\right)$ to $W^{1, q}\left(D_{L} \cup T_{L}\right)$, for all $q \in[1,+\infty)$ : there is a positive constant c (depending on $q$ ) such that, for all $u \in W^{1, q}\left(D_{L}\right),\left\|\mathcal{E}_{L} u\right\|_{W^{1, q}\left(T_{L}\right)} \leq$ $\mathrm{c}\|u\|_{W^{1, q}\left(D_{L}\right)}$.

Call $\Omega_{L}^{0}=\Omega^{0} \cap \bigcup_{n \in \mathbb{N}} F_{1}^{n}\left(D_{L}\right)$ and $\widetilde{\Omega}_{L}^{0}=\widetilde{\Omega}^{0} \cap \bigcup_{n \in \mathbb{N}} F_{1}^{n}\left(D_{L} \cup T_{L}\right)$. Similarly $\Omega_{R}^{0}=\Omega^{0} \cap \bigcup_{n \in \mathbb{N}} F_{2}^{n}\left(D_{R}\right)$ and $\widetilde{\Omega}_{R}^{0}=\widetilde{\Omega}^{0} \cap \bigcup_{n \in \mathbb{N}} F_{2}^{n}\left(D_{R} \cup T_{R}\right)$. The previous observation and the facts that

- $\left|F_{1}^{n}\left(T_{L}\right) \cap F_{1}^{m}\left(T_{L}\right)\right|=0$ if $n \neq m$;

- for any point $x$ in $\Omega_{L}^{0}$, there exists at least one and at most two integers $n$ such that $x \in F_{1}^{n}\left(D_{L}\right)$, enable to construct an extension operator $\widetilde{\mathcal{E}}_{L}$, bounded from $W^{1, q}\left(\Omega_{L}^{0}\right)$ to $W^{1, q}\left(\widetilde{\Omega}_{L}^{0}\right), 1 \leq q<\infty$, by:

$$
\begin{aligned}
& \text { if } x \in F_{1}^{n}\left(T_{L}\right), n \geq 1 \text {, then }\left(\widetilde{\mathcal{E}}_{L} u\right)(x)=\mathcal{E}_{L}\left(\left.\left(u \circ F_{1}^{n}\right)\right|_{D_{L}}\right)\left(\left(F_{1}^{n}\right)^{-1}(x)\right) \text {, } \\
& \text { if } x \in \widetilde{\Omega}^{0} \cap T_{L} \text {, then }\left(\widetilde{\mathcal{E}}_{L} u\right)(x)=\mathcal{E}_{L, 0}\left(\left.u\right|_{D_{L}}\right)(x),
\end{aligned}
$$

where $\mathcal{E}_{L, 0}$ is any extension operator which maps continuously $W^{1, q}\left(\Omega^{0} \cap D_{L}\right)$ to $W^{1, q}\left(\widetilde{\Omega}^{0} \cap\left(D_{L} \cup T_{L}\right)\right)$, $1 \leq q<\infty$.

By symmetry, it is possible to construct an extension operator $\widetilde{\mathcal{E}}_{R}$, bounded from $W^{1, q}\left(\Omega_{R}^{0}\right)$ to $W^{1, q}\left(\widetilde{\Omega}_{R}^{0}\right)$, $1 \leq q<\infty$. 
Second step. Let $\mathcal{G}_{L}=\left\{F_{2} \circ \mathcal{M}_{\sigma}\left(F_{1}, F_{2}\right), \sigma \in \mathcal{A}_{n}, n \in \mathbb{N}\right\}$ and $\mathcal{G}_{R}=\left\{F_{1} \circ \mathcal{M}_{\sigma}\left(F_{1}, F_{2}\right), \sigma \in \mathcal{A}_{n}, n \in \mathbb{N}\right\}$. We observe that for any point $x$ in $\widetilde{\Omega}^{0} \backslash \Omega^{0}$, one and only one of the following four conditions is true:

(1) either $x \in \widetilde{\Omega}_{L}^{0} \backslash \Omega^{0}$;

(2) or $x \in \widetilde{\Omega}_{R}^{0} \backslash \Omega^{0}$;

(3) or there exists a unique transformation $\tau$ in $\mathcal{G}_{L}$ such that $x \in \tau\left(\widetilde{\Omega}_{L}^{0} \backslash \Omega^{0}\right)$;

(4) or there exists a unique transformation $\tau$ in $\mathcal{G}_{R}$ such that $x \in \tau\left(\widetilde{\Omega}_{R}^{0} \backslash \Omega^{0}\right)$.

From this observation, it is possible to construct an extension operator $\mathcal{E}$, bounded from $W^{1, q}\left(\Omega^{0}\right)$ to $W^{1, q}\left(\widetilde{\Omega}^{0}\right)$, $1 \leq q<\infty$, by: for $x \in \widetilde{\Omega}^{0} \backslash \Omega^{0}$,

- if condition 1 is true: $(\mathcal{E} u)(x)=\left(\widetilde{\mathcal{E}}_{L} u\right)(x)$;

- if condition 2 is true: $(\mathcal{E} u)(x)=\left(\widetilde{\mathcal{E}}_{R} u\right)(x)$;

- if condition 3 is true: $(\mathcal{E} u)(x)=\left(\widetilde{\mathcal{E}}_{L}(u \circ \tau)\right)\left(\tau^{-1}(x)\right)$;

- if condition 4 is true: $(\mathcal{E} u)(x)=\left(\widetilde{\mathcal{E}}_{R}(u \circ \tau)\right)\left(\tau^{-1}(x)\right)$.

\subsection{Bounded extension from $W^{1, q}\left(\widetilde{\Omega}^{0}\right)$ to $W^{1, q}\left(\widehat{\Omega}^{0}\right), 1 \leq q<2$}

Lemma 11. There exists an extension operator $\mathcal{F}$ bounded from $W^{1, q}\left(\widetilde{\Omega}^{0}\right)$ to $W^{1, q}\left(\widehat{\Omega}^{0}\right)$, for all $q, 1 \leq q<2$.

Proof. Consider the triangle $\widehat{T}$ whose vertices are $(0,6),\left(\frac{3}{2}, 2\right)$, and $\left.\left(-\frac{3}{2}, 2\right)\right)(\widehat{T}$ is displayed on the left of Figure 3 with interrupted lines). The key observation is that $\widehat{T} \backslash T \subset \widetilde{\Omega}^{0}$, and that

$$
\forall \sigma_{1}, \sigma_{2} \in \bigcup_{n \in \mathbb{N}} \mathcal{A}_{n} \text { such that } \sigma_{1} \neq \sigma_{2}, \quad \mathcal{M}_{\sigma_{1}}\left(F_{1}, F_{2}\right)(\widehat{T}) \cap \mathcal{M}_{\sigma_{2}}\left(F_{1}, F_{2}\right)(\widehat{T})=\emptyset .
$$

By using known results on Sobolev spaces on polygonal domains, see e.g. [7], there exists a extension operator $\widetilde{\mathcal{F}}$, bounded from $W^{1, q}(\widehat{T} \backslash T)$ to $W^{1, q}(\widehat{T})$, for any $q, 1 \leq q<2$. Note that the operator cannot be bounded from $W^{1,2}(\widehat{T} \backslash T)$ to $W^{1,2}(\widehat{T})$, see [7]. by

From this and (89), we can construct an extension operator $\mathcal{F}$, bounded from $W^{1, q}\left(\widetilde{\Omega}^{0}\right)$ to $W^{1, q}\left(\widehat{\Omega}^{0}\right), 1 \leq q<2$

$$
\text { if } x \in \mathcal{M}_{\sigma}\left(F_{1}, F_{2}\right)(T), \quad \mathcal{F}(u)(x)=\widetilde{\mathcal{F}}\left(\left.\left(u \circ \mathcal{M}_{\sigma}\left(F_{1}, F_{2}\right)\right)\right|_{\widehat{T} \backslash T}\right) \circ\left(\mathcal{M}_{\sigma}\left(F_{1}, F_{2}\right)\right)^{-1}(x) \text {. }
$$

\subsection{Bounded extension from $W^{1, q}\left(\Omega^{0}\right)$ to $W^{1, q}\left(\widehat{\Omega}^{0}\right), 1 \leq q<2$}

The operator $\mathcal{J}=\mathcal{F} \circ \mathcal{E}$ is an extension operator bounded from $W^{1, q}\left(\Omega^{0}\right)$ to $W^{1, q}\left(\widehat{\Omega}^{0}\right), 1 \leq q<2$. Theorem 3 is proved.

\section{Proof of Theorem 9}

To prove Theorem 9, we need to study the stability of the inductive construction of $T_{k}^{0, r}$ in Algorithm 3 with respect to $Z^{0}$ :

Proposition 8. Let $X_{q}$ be a sequence of operators in $\mathbb{O}$ converging to $T^{0}$ as $q \rightarrow \infty$. For an integer $r$, and $0 \leq j \leq r$, let us call $Z_{q, r}^{j}$ the operators such that

- $Z_{q, r}^{0}=X_{q}$

- for $0 \leq j<r, Z_{q, r}^{j+1}$ is obtained from $Z_{q, r}^{j}$ by the induction (I.F.) above.

Assume that $k \notin S p^{0, r}$. Then, there exists an integer $Q$ such that for all $q>Q, Z_{q, r}^{r}$ is a bounded operator from $H^{\frac{1}{2}}\left(\Gamma^{0}\right)$ to its dual, and for $q>Q$, for a constant $\mathrm{C}$ independent on $q$,

$$
\left\|Z_{q, r}^{r}-T_{k}^{0, r}\right\| \leq \mathrm{C}\left\|X_{q}-T^{0}\right\| .
$$


Proof. Since $k \notin S p^{0, r}$, the problem: find $u_{g} \in \mathcal{V}\left(Y^{r-1}\right)$, such that for all $v \in \mathcal{V}\left(Y^{r-1}\right)$,

$$
\int_{Y^{r-1}} \nabla u_{g} \cdot \nabla v-k \int_{Y^{r-1}} u_{g} v+\sum_{\sigma \in \mathcal{A}_{r}}\left\langle T^{0}\left(\left.u_{g}\right|_{\Gamma^{\sigma}} \circ \mathcal{M}_{\sigma}\left(F_{1}, F_{2}\right)\right),\left.v\right|_{\Gamma^{\sigma}} \circ \mathcal{M}_{\sigma}\left(F_{1}, F_{2}\right)\right\rangle=\langle g, v\rangle
$$

defines an isomorphism $\Psi: g \mapsto u_{g}$ from $\mathcal{V}^{\prime}\left(Y^{r-1}\right)$ onto $\mathcal{V}\left(Y^{r-1}\right)$.

For $u \in H^{\frac{1}{2}}\left(\Gamma^{0}\right)$, consider the problem: find $\tilde{u} \in H^{1}\left(Y^{r-1}\right)$ such that $\left.\tilde{u}\right|_{\Gamma^{0}}=u$ and for all $v \in \mathcal{V}\left(Y^{r-1}\right)$,

$$
\int_{Y^{r-1}} \nabla \tilde{u} \cdot \nabla v-k \int_{Y^{r-1}} \tilde{u} v+\sum_{\sigma \in \mathcal{A}_{r}}\left\langle X_{q}\left(\left.\tilde{u}\right|_{\Gamma^{\sigma}} \circ \mathcal{M}_{\sigma}\left(F_{1}, F_{2}\right)\right),\left.v\right|_{\Gamma^{\sigma}} \circ \mathcal{M}_{\sigma}\left(F_{1}, F_{2}\right)\right\rangle=0 .
$$

Let $\hat{u}=\mathcal{H}_{k}^{0, r} u$. Problem (91) is equivalent to finding $e=\tilde{u}-\hat{u} \in \mathcal{V}\left(Y^{r-1}\right)$ such that for all $v \in \mathcal{V}\left(Y^{r-1}\right)$,

$$
\begin{gathered}
\int_{Y^{r-1}} \nabla e \cdot \nabla v-k \int_{Y^{r-1}} e v+\sum_{\sigma \in \mathcal{A}_{r}}\left\langle T^{0}\left(\left.e\right|_{\Gamma^{\sigma}} \circ \mathcal{M}_{\sigma}\left(F_{1}, F_{2}\right)\right),\left.v\right|_{\Gamma^{\sigma}} \circ \mathcal{M}_{\sigma}\left(F_{1}, F_{2}\right)\right\rangle \\
=\sum_{\sigma \in \mathcal{A}_{r}}\left\langle\left(T^{0}-X_{q}\right)\left(\left.(e+\hat{u})\right|_{\Gamma^{\sigma}} \circ \mathcal{M}_{\sigma}\left(F_{1}, F_{2}\right)\right),\left.v\right|_{\Gamma^{\sigma}} \circ \mathcal{M}_{\sigma}\left(F_{1}, F_{2}\right)\right\rangle,
\end{gathered}
$$

so (91) can be reformulated as a fixed point problem with a linear operator involving $\Psi$. For $q$ large enough, the operator in the fixed point is a contraction, so (91) has a unique solution and there exists $Q>0$ and a constant $\mathrm{C}>0$ such that for all $q>Q$

$$
\|\hat{u}-\tilde{u}\|_{H^{1}\left(Y^{r-1}\right)} \leq \mathrm{C}\left\|T^{0}-X_{q}\right\|\|u\|_{H^{\frac{1}{2}}\left(\Gamma^{0}\right)} .
$$

Now, one can check that, for all $v \in H^{1}\left(Y^{r-1}\right)$,

$$
\left\langle Z_{q, r}^{r} u,\left.v\right|_{\Gamma^{0}}\right\rangle=\int_{Y^{r-1}} \nabla \tilde{u} \cdot \nabla v-k \int_{Y^{r-1}} \tilde{u} v+\sum_{\sigma \in \mathcal{A}_{r}}\left\langle X_{q}\left(\left.\tilde{u}\right|_{\Gamma^{\sigma}} \circ \mathcal{M}_{\sigma}\left(F_{1}, F_{2}\right)\right),\left.v\right|_{\Gamma^{\sigma}} \circ \mathcal{M}_{\sigma}\left(F_{1}, F_{2}\right)\right\rangle,
$$

so from (79) and (78) in the case $n=r,(93)$ and (92), one can deduce (90).

Theorem 9 is a consequence of Theorem 7 , Lemma 8 and Proposition 8.

Acknowledgements. The research of the first and third authors was partially supported by the project ACI Nouvelles Interfaces des Mathématiques "LePoumonVousDisJe".

\section{REFERENCES}

[1] Y. Achdou, C. Sabot and N. Tchou, A multiscale numerical method for Poisson problems in some ramified domains with a fractal boundary. SIAM Multiscale Model. Simul. (2006) (accepted for publication).

[2] Y. Achdou, C. Sabot and N. Tchou, Transparent boundary conditions for Helmholtz equation in some ramified domains with a fractal boundary. J. Comput. Phys. (2006) (in press).

[3] R.A. Adams, Sobolev spaces. Academic Press, New York-London (1975). Pure Appl. Math. 65.

[4] H. Brezis, Analyse fonctionnelle. Collection Mathématiques Appliquées pour la Maîtrise. Théorie et applications. Masson, Paris, 1983.

[5] M. Felici, Physique du transport diffusif de l'oxygène dans le poumon humain. Ph.D. thesis, École Polytechnique (2003).

[6] M. Gibbons, A. Raj and R.S. Strichartz, The finite element method on the Sierpinski gasket. Constr. Approx. 17 (2001) $561-588$.

[7] P. Grisvard, Elliptic problems in nonsmooth domains. Monographs and Studies in Mathematics 24, Pitman (Advanced Publishing Program), Boston, MA (1985).

[8] J.E. Hutchinson, Fractals and self-similarity. Indiana Univ. Math. J. 30 (1981) 713-747. 
[9] P.W. Jones, Quasiconformal mappings and extendability of functions in Sobolev spaces. Acta Math. 147 (1981) 71-88.

[10] A. Jonsson and H. Wallin, Function spaces on subsets of $\mathbf{R}^{n}$. Math. Rep. 2 (1984) xiv+221.

[11] J.B. Keller and D. Givoli, Exact nonreflecting boundary conditions. J. Comput. Phys. 82 (1989) 172-192.

[12] M.R. Lancia, A transmission problem with a fractal interface. Z. Anal. Anwendungen 21 (2002) 113-133.

[13] M.R. Lancia, Second order transmission problems across a fractal surface. Rend. Accad. Naz. Sci. XL Mem. Mat. Appl. (5) 27 (2003) 191-213.

[14] B.B. Mandelbrodt, The fractal geometry of nature. Freeman and Co (1982).

[15] B. Mauroy, M. Filoche, J.S. Andrade and B. Sapoval, Interplay between flow distribution and geometry in an airway tree. Phys. Rev. Lett. 90 (2003).

[16] B. Mauroy, M. Filoche, E.R. Weibel and B. Sapoval, The optimal bronchial tree is dangerous. Nature 427 (2004) $633-636$.

[17] V.G. Maz'ja, Sobolev spaces. Springer Series in Soviet Mathematics. Springer-Verlag, Berlin (1985). Translated from the Russian by T.O. Shaposhnikova.

[18] U. Mosco, Energy functionals on certain fractal structures. J. Convex Anal. 9 (2002) 581-600.

[19] U. Mosco and M.A. Vivaldi, Variational problems with fractal layers. Rend. Accad. Naz. Sci. XL Mem. Mat. Appl. (2003) 237-251.

[20] R. Oberlin, B. Street and R.S. Strichartz, Sampling on the Sierpinski gasket. Experiment. Math. 12 (2003) $403-418$.

[21] J. Rauch, Partial differential equations. Graduate Texts in Mathematics 128, Springer-Verlag, New York (1991).

[22] C. Sabot, Spectral properties of self-similar lattices and iteration of rational maps. Mém. Soc. Math. Fr. (N.S.) 92 (2003) vi+104.

[23] C. Sabot, Electrical networks, symplectic reductions, and application to the renormalization map of self-similar lattices, in Fractal geometry and applications: a jubilee of Benoît Mandelbrot. Part 1, Proc. Sympos. Pure Math., Amer. Math. Soc., Providence, RI, 72 (2004) 155-205.

[24] B. Sapoval and T. Gobron, Vibration of strongly irregular fractal resonators. Phys. Rev. E 47 (1993).

[25] B. Sapoval, T. Gobron and A. Margolina, Vibration of fractal drums. Phys. Rev. Lett. 67 (1991).

To access this journal online:

www.edpsciences.org 